\title{
Pump-driven normal-to-excitonic insulator transition: Josephson oscillations and signatures of BEC-BCS crossover in time-resolved ARPES
}

\author{
E. Perfetto, ${ }^{1,2,3}$ D. Sangalli, ${ }^{2}$ A. Marini, ${ }^{2}$ and G. Stefanucci $\odot^{1,3}$ \\ ${ }^{1}$ Dipartimento di Fisica, Università di Roma Tor Vergata, Via della Ricerca Scientifica 1, 00133 Rome, Italy \\ ${ }^{2}$ Istituto di Struttura della materia (ISM-CNR), Division of Ultrafast Processes in Materials (FLASHit), Via Salaria km 29.3, Area della \\ Ricerca di Roma 1, 0016 Monterotondo Scalo, Italy \\ ${ }^{3}$ INFN, Sezione di Roma Tor Vergata, Via della Ricerca Scientifica 1, 00133 Rome, Italy
}

(Received 13 June 2019; revised manuscript received 18 October 2019; published 16 December 2019)

\begin{abstract}
We consider a ground-state band insulator turning into a nonequilibrium excitonic insulator (NEQ-EI) upon visiting properly selected and physically relevant highly excited states. The NEQ-EI phase, characterized by self-sustained oscillations of the complex order parameter, neatly follows from a nonequilibrium Green's function treatment on the Konstantinov-Perel' contour. We present the first $a b$ initio band structure of LiF, a ground-state bulk insulator, in different NEQ-EI states. We also show that NEQ-EI states can be generated by currently available pump pulses. Peculiar fingerprints of the NEQ-EI phase in time-resolved angle-resolved photoemission spectroscopy spectra are highlighted: (i) during the pump-driving, the system goes through a BEC-BCS crossover and (ii) concomitantly the excitonic spectral structure undergoes a convex-to-concave shape transition; (iii) attosecond pulses shone after the pump-driving at different times $t_{\text {delay }}$ produce a photocurrent that oscillates in $t_{\text {delay }}$ with a pump-tunable frequency-we relate this phenomenon to the ac response of an exotic Josephson junction.
\end{abstract}

DOI: 10.1103/PhysRevMaterials.3.124601

\section{INTRODUCTION}

At low enough temperature, small-gap insulators, semimetals, as well as metals with overlapping bands may turn into excitonic insulators (EIs) due to the Coulomb electronhole attraction [1-7]. Theoretical and experimental works on different materials, e.g., quantum Hall bilayers [8,9], graphene bilayers [10-13], carbon nanotubes [14,15], and chalcogenide-based structures [16-20], have addressed signatures and properties of this equilibrium EI phase, including dynamical responses to external laser pulses [21-26] and adiabatic switching of electronic correlations [27]. One of the most interesting signatures of the EI phase is the flattening of the valence band, recently revealed in $\mathrm{Ta}_{2} \mathrm{NiSe}_{5}$ using angleresolved photoemission spectroscopy (ARPES) $[28,29]$.

The past decade has seen a renewed interest in systems that are semimetals [30,31] or insulators [32-38] in the ground state but exhibit an EI phase in some (possibly pump-induced) nonequilibrium (NEQ) excited state. The optical properties of a NEQ-EI have been calculated by several authors in the past [39-42] and were recently measured in bulk GaAs [43]. However, it was not until 1993 that Östreichand and Schönhammer pointed out a crucial difference between a ground-state EI and a NEQ-EI [44]: in a NEQ-EI, the macroscopic polarization after the pump has been switched off exhibits persistent, self-sustained oscillations with a finite, time-independent amplitude and a frequency that depends on the absorbed energy. A similar dynamical excited state of matter has been independently shown to emerge in mean-field approximations using an ansatz for the time-dependent order parameter [32]. Here we provide an alternative derivation based on nonequilibrium Green's functions (NEGFs) and show the equivalence between the pump-induced state [44] and the dynamical excited state [32].

The stability of the NEQ-EI phase plays of course a crucial role in applications to, e.g., optoelectronics [45]. Hanai et al. have pointed out the existence of regimes for which small fluctuations of the order parameter destroy the phase (dynamical instability) [34], and they also studied the effects of exciton-boson coupling [33]. Hannewald et al. studied the NEQ-EI lifetime due to dephasing [46]. A beyond-mean-field analysis including light-matter interaction and damping has confirmed the existence of the NEQ-EI phase [36]. Real-time first-principles studies are foreseeable in the near future for quantitative, material-dependent predictions.

In this work, we apply the NEGF formalism to systems that exhibit a NEQ-EI phase but are normal band insulators (BIs) in the ground state, and we highlight the NEQ-EI fingerprints visible in ARPES spectra. We first show the existence of optimal pump pulses with subgap frequency (slightly offresonance with respect to the single-exciton energy), which generate the same dynamical excited state [44] as an (excited) self-consistent calculation. The proposed self-consistent theory is also implemented in the ab-initio YАMBO code [47], and the spectral function of a LiF bulk insulator in the NEQEI phase is presented and discussed. Our main findings are as follows: (i) the system undergoes a BEC-BCS crossover $[48,49]$ while driving it with the optimal pump-pulses; (ii) during the BEC-BCS crossover, a convex-to-concave shape transition in the excitonic structure of time-resolved ARPES spectra occurs; (iii) the photocurrent generated by attosecond 
pulses shone at different times $t_{\text {delay }}$ oscillates in $t_{\text {delay }}$ with a frequency that is larger than both the optimal pump frequency and the exciton energy: we relate this phenomenon to the ac response of an exotic Josephson junction.

The paper is organized as follows. In Sec. II we illustrate the self-consistent NEGF approach to excited-state systems and derive the equations to calculate the Green's function. In Sec. III we discuss the NEQ-EI phase diagram of a two-band model Hamiltonian. We determine the critical conduction density at the BEC-BCS crossover and the boundary between the BI and NEQ-EI phases. We further illustrate how the NEQ spectral function changes in these different regimes. The simplifications of the two-band model are relaxed in Sec. IV, where we present the spectral function of a $\mathrm{LiF}$ bulk insulator in the NEQ-EI phase. In Sec. V we perform real-time simulations and obtain the optimal pump pulse that brings the system closest to the self-consistent NEGF results. The time-resolved ARPES spectrum is calculated in Sec. VI for different probe durations, highlighting the signatures of the BEC-BCS crossover and the Josephson-like oscillations. A summary of the main results and concluding remarks are contained in Sec. VII.

\section{MODEL HAMILTONIAN AND EXCITED-STATE GREEN'S FUNCTION}

To describe the main qualitative aspects of the NEQ-EI phase, we initially discard several complications of realistic materials. We consider a one-dimensional spinless insulator with one valence band and one conduction band separated by a direct gap of magnitude $\epsilon_{g}$, and we assume that the intraband repulsion between the electrons is negligible. A similar model has been proposed in Ref. [50] to discuss excitons within the time-dependent density functional theory (TDDFT) and more recently in Refs. [21-23] to drive out-of-equilibrium systems that are EI in the ground state. In Sec. IV we apply the NEGF theory to LiF and relax all the simplifications of the model; multiple bands and valleys, intraband and interband repulsion, band anisotropies and degeneracies, as well as spin-exchange effects will all be taken into account.

The explicit form of the model Hamiltonian reads

$$
\begin{aligned}
\hat{H}= & \sum_{k}\left(\epsilon_{v k} \hat{v}_{k}^{\dagger} \hat{v}_{k}+\epsilon_{c k} \hat{c}_{k}^{\dagger} \hat{c}_{k}\right)-U_{0} \sum_{k} \hat{c}_{k}^{\dagger} \hat{c}_{k} \\
& +\frac{1}{\mathcal{N}} \sum_{k_{1} k_{2} q} U_{q} \hat{v}_{k_{1}+q}^{\dagger} \hat{c}_{k_{2}-q}^{\dagger} \hat{c}_{k_{2}} \hat{v}_{k_{1}},
\end{aligned}
$$

where $\hat{v}_{k}\left(\hat{c}_{k}\right)$ annihilates an electron of momentum $k$ in the valence (conduction) band, $U_{q}$ is the Coulomb interaction, and $\mathcal{N}$ is the number of discretized $k$-points. As we have ignored the intraband interaction, the equilibrium Hartree potential is zero for valence electrons and it is equal to $U$ for conduction electrons. Consistently, the positive background interacts only with the conduction electrons; see the last term in the first row.

The Hamiltonian in Eq. (1) is invariant under the "local" gauge transformation $\hat{c} \rightarrow e^{i \theta} \hat{c}$ for the conduction-band operators. This gauge symmetry is broken by any state with a nonvanishing average $\left\langle\hat{c}_{k}^{\dagger} \hat{v}_{k}\right\rangle$ since under a gauge transformation $\left\langle\hat{c}_{k}^{\dagger} \hat{v}_{k}\right\rangle \rightarrow e^{-i \theta}\left\langle\hat{c}_{k}^{\dagger} \hat{v}_{k}\right\rangle$. The conserved quantity associated with this gauge symmetry is the number of electrons $N_{c}$ in the conduction band (hence the number of electrons $N_{v}$ in the valence band is conserved as well). Each many-body eigenstate is then characterized by a well-defined number of electrons $N_{c}$ and $N_{v}$. A nonvanishing $\left\langle\hat{c}_{k}^{\dagger} \hat{v}_{k}\right\rangle$ is therefore possible only provided that several many-body eigenstates with different $N_{c}$ and $N_{v}$, constrained by $\left(N_{c}+N_{v}\right) / \mathcal{N}=1$, become degenerate in the thermodynamic limit. This can happen for the ground-state multiplet (in this case, the exciton energy $\epsilon_{\mathrm{x}} \lesssim 0$ ) and/or for excited-state multiplets. In this work, we are especially interested in the latter scenario.

We consider $\epsilon_{g} / U_{0}$ large enough for the exciton energy to satisfy $0 \ll \epsilon_{\mathrm{x}} \lesssim \epsilon_{g}$. Then, the ground state is nondegenerate and it consists of a fully occupied valence band and a completely empty conduction band (consequently the groundstate average of the interaction with the positive background vanishes). To investigate the existence of a NEQ-EI phase, we use the NEGF formalism. In NEGF the fundamental quantity is the Keldysh-Green's function, which for our model Hamiltonian reads [51]

$$
G_{k}^{\alpha \beta}\left(z, z^{\prime}\right)=\frac{1}{i} \frac{\operatorname{Tr}\left[\mathcal{T}\left\{e^{-i \int d \bar{z} \hat{H}(\bar{z})} \hat{\psi}_{\alpha k}(z) \hat{\psi}_{\beta k}^{\dagger}\left(z^{\prime}\right)\right\}\right]}{\operatorname{Tr}\left[\mathcal{T}\left\{e^{-i \int d \bar{z}(\bar{z})}\right\}\right]},
$$

where $\hat{\psi}_{\alpha k}=\hat{v}_{k}, \hat{c}_{k}$ for $\alpha=v, c$. In Eq. (2) the arguments $z, z^{\prime}$ as well as the integral over $\bar{z}$ run on the KonstantinovPerel' contour [52] consisting of a forward and backward branch $(0, \infty) \cup(\infty, 0)$ joined to a vertical imaginary track $(0,-i \beta)$, with $\beta$ the inverse temperature; $\mathcal{T}$ is the contour ordering operator. In the absence of external fields, $\hat{H}(z)=\hat{H}$ for $z$ on the forward or backward branches. If the system is initially in thermal equilibrium at chemical potential $\mu$, then the Hamiltonian on the imaginary track is $\hat{H}(z) \equiv \hat{H}^{\mathrm{M}}=\hat{H}-$ $\mu \hat{N}$, with $\hat{N}=\hat{N}_{v}+\hat{N}_{c}$ and $\hat{N}_{\alpha} \equiv \sum_{k} \hat{\psi}_{\alpha k}^{\dagger} \hat{\psi}_{\alpha k}$ the number operator for electrons in band $\alpha$. This choice of $\hat{H}^{\mathrm{M}}$ does indeed correspond to averaging with the thermal equilibrium density matrix $\hat{\rho}=e^{-\beta(\hat{H}-\mu \hat{N})} / \mathcal{Z}, \mathcal{Z}$ being the partition function. The resulting $G_{k}^{\alpha \beta}\left(z, z^{\prime}\right)$ in Eq. (2) is the Matsubara Green's function for both $z$ and $z^{\prime}$ on the vertical track and the equilibrium real-time Green's function for $z$ and $z^{\prime}$ on the forward or backward branches [51].

To calculate the Green's function in some excited state, we must average with an excited density matrix $\hat{\rho}=e^{-\beta \hat{H}^{\mathrm{M}}} / \mathcal{Z}$, where $\hat{H}^{\mathrm{M}}$ is a self-adjoint operator with the property $\left[\hat{H}^{\mathrm{M}}, \hat{H}\right]=0$. Here we consider

$$
\hat{H}^{\mathrm{M}}=\hat{H}-\mu_{v} \hat{N}_{v}-\mu_{c} \hat{N}_{c},
$$

with $\mu_{v} \neq \mu_{c}$. This $\hat{H}^{\mathrm{M}}$ commutes with $\hat{H}$ since $\left[\hat{N}_{\alpha}, \hat{H}\right]=0$. For $U_{q}=0$ and zero temperature, averaging with $\hat{\rho}$ is equivalent to averaging over a state with the valence- (conduction-) band populated up to the chemical potential $\mu_{v}\left(\mu_{c}\right)$. Hereafter, we use $\hat{H}^{\mathrm{M}}$ in Eq. (3) for the Hamiltonian on the imaginary track.

The exact equation of motion for the Green's function in Eq. (2) reads (in a $2 \times 2$ matrix form)

$$
\left[i \frac{d}{d z}-h_{k}(z)\right] G_{k}\left(z, z^{\prime}\right)=\delta\left(z, z^{\prime}\right)+\int d \bar{z} \Sigma_{k}(z, \bar{z}) G_{k}\left(\bar{z}, z^{\prime}\right),
$$


where $\Sigma_{k}$ is the sum of the Hartree-Fock (HF) and correlation self-energies, and $h_{k}(z)$ is given by

$$
\begin{aligned}
h_{k}(z) & =\left(\begin{array}{cc}
\epsilon_{v k} & 0 \\
0 & \epsilon_{c k}-U_{0}
\end{array}\right)-\theta_{\mid}(z)\left(\begin{array}{cc}
\mu_{v} & 0 \\
0 & \mu_{c}
\end{array}\right) \\
& \equiv h_{k}-\theta_{\mid}(z) m
\end{aligned}
$$

with $\theta_{\mid}(z)=0$ if $z$ is on the forward or backward branches and $\theta_{\mid}(z)=1$ if $z$ is on the imaginary track. In the Hartree-Fock (HF) approximation, we have $\Sigma_{k}^{\alpha \beta}\left(z, z^{\prime}\right)=\delta\left(z, z^{\prime}\right) V_{k}^{\alpha \beta}(z)$, where the HF potential reads

$$
\begin{aligned}
V_{k}^{\alpha \alpha}(z) & =-\frac{i}{\mathcal{N}} \sum_{q} U_{0} G_{q}^{\bar{\alpha} \bar{\alpha}}\left(z, z^{+}\right), \\
V_{k}^{\alpha \bar{\alpha}}(z) & =\frac{i}{\mathcal{N}} \sum_{q} U_{k-q} G_{q}^{\alpha \bar{\alpha}}\left(z, z^{+}\right),
\end{aligned}
$$

with $\bar{\alpha}=c, v$ for $\alpha=v, c$, and $z^{+}$is the contour time infinitesimally later than $z$. As we have discarded the intraband interaction, the diagonal elements of $V$ are only due to the Hartree diagram, whereas the off-diagonal ones are only due to the exchange (Fock) diagram. This simplification is relaxed in Sec. IV.

\section{A. Matsubara Green's function}

Choosing $z=-i \tau$ and $z^{\prime}=-i \tau^{\prime}$ on the imaginary track, we get the Matsubara Green's function $G_{k}^{\mathrm{M}}\left(\tau, \tau^{\prime}\right) \equiv$ $G_{k}\left(-i \tau,-i \tau^{\prime}\right)$. Since the HF self-energy is local in time, the structure of $G^{\mathrm{M}}$ is the same as in the noninteracting case, the only difference being that the one-body noninteracting matrix

$$
h_{k}-m \equiv h_{k}^{\mathrm{M}},
$$

see Eq. (5), is replaced by the one-body HF matrix $h_{k}^{\mathrm{M}}+V_{k}$, with $V_{k} \equiv V_{k}(-i \tau)$ the HF potential on the imaginary track [51]. Therefore,

$$
\begin{aligned}
G_{k}^{\mathrm{M}}\left(\tau, \tau^{\prime}\right)= & -i\left[\theta\left(\tau, \tau^{\prime}\right) \bar{f}\left(h_{k}^{\mathrm{M}}+V_{k}\right)-\theta\left(\tau^{\prime}, \tau\right)\right. \\
& \left.\times f\left(h_{k}^{\mathrm{M}}+V_{k}\right)\right] e^{-\left(\tau-\tau^{\prime}\right)\left(h_{k}^{\mathrm{M}}+V_{k}\right)},
\end{aligned}
$$

where $f(\omega)=1 /\left(e^{\beta \omega}+1\right)$ is the Fermi function and $\bar{f}(\omega)=$ $1-f(\omega)$. As $V_{k}$ depends on $G_{k}^{\mathrm{M}}$, see Eqs. (6) and (7), the problem has to be solved self-consistently. We point out that for the Matsubara Green's function to be correctly antiperiodic in $\tau$ and $\tau^{\prime}$ with period $\beta$, the population $n_{\alpha k}=$ $-i G_{k}^{\alpha \alpha, \mathrm{M}}\left(\tau, \tau^{+}\right)$of band $\alpha=v, c$ cannot be an input of the self-consistency cycle.

Let $e_{k}^{\lambda}$ and $\varphi_{k}^{\lambda}=\left(\begin{array}{c}\varphi_{v k}^{\lambda} \\ \varphi_{c k}^{\lambda}\end{array}\right), \lambda= \pm$, be the two eigenvalues and eigenvectors of $h_{k}^{\mathrm{M}}+V_{k}$. Without any loss of generality, we choose $\varphi_{\alpha k}^{\lambda}$ real. Then, from Eq. (9),

$$
G_{k}^{\alpha \beta, \mathrm{M}}\left(\tau, \tau^{+}\right)=i \sum_{\lambda} f\left(e_{k}^{\lambda}\right) \varphi_{\alpha k}^{\lambda} \varphi_{\beta k}^{\lambda},
$$

and therefore the matrix elements of the HF potential in Eqs. (6) and (7) can be rewritten as

$$
V_{k}^{\alpha \alpha}=\frac{1}{\mathcal{N}} \sum_{q} U_{0} \sum_{\lambda} f\left(e_{q}^{\lambda}\right)\left|\varphi_{\bar{\alpha} q}^{\lambda}\right|^{2},
$$

$$
V_{k}^{\alpha \bar{\alpha}}=-\frac{1}{\mathcal{N}} \sum_{q} U_{k-q} \sum_{\lambda} f\left(e_{q}^{\lambda}\right) \varphi_{\alpha q}^{\lambda} \varphi_{\bar{\alpha} q}^{\lambda} .
$$

In terms of the quantities

$$
\begin{gathered}
\tilde{\epsilon}_{v k} \equiv \epsilon_{v k}+V_{k}^{v v}-\mu_{v}, \\
\tilde{\epsilon}_{c k} \equiv \epsilon_{c k}-U_{0}+V_{k}^{c c}-\mu_{c}, \\
\Delta_{k} \equiv V_{k}^{c v}=V_{k}^{v c},
\end{gathered}
$$

it is straightforward to find the eigenvalues

$$
e_{k}^{\lambda}=\frac{\tilde{\epsilon}_{v k}+\tilde{\epsilon}_{c k}+\lambda R}{2},
$$

with $R=\sqrt{\left(\tilde{\epsilon}_{v k}-\tilde{\epsilon}_{c k}\right)^{2}+4 \Delta_{k}^{2}}$, and the eigenvectors

$$
\varphi_{k}^{\lambda}=\left(\begin{array}{c}
\lambda \sqrt{\frac{1}{2}\left(1+\lambda \frac{\tilde{\epsilon}_{v k}-\tilde{\epsilon}_{c k}}{R}\right)} \\
\operatorname{sgn}\left(\Delta_{k}\right) \sqrt{\frac{1}{2}\left(1-\lambda \frac{\tilde{\epsilon}_{v k}-\tilde{\epsilon}_{c k}}{R}\right)}
\end{array}\right),
$$

which when inserted in Eqs. (11) and (12) provide a closed system of equations for the HF potential. Henceforth we refer to $\Delta_{k}$ as the order parameter since a nonvanishing $\Delta_{k}$ implies that $\left\langle\hat{c}_{q}^{\dagger} \hat{v}_{q}\right\rangle \neq 0$ at least for some $q$, and hence a spontaneous symmetry breaking.

\section{B. Real-time Green's function}

Once the Matsubara Green's function is known, we can calculate the Green's function with both arguments on the real axis. In the HF approximation, the lesser Green's function, which solves Eq. (4), reads [51]

$$
G_{k}^{\alpha \beta,<}\left(t, t^{\prime}\right)=i \sum_{\lambda} f\left(e_{k}^{\lambda}\right) \varphi_{\alpha k}^{\lambda}(t) \varphi_{\beta k}^{\lambda *}\left(t^{\prime}\right),
$$

where the time-dependent vectors satisfy

$$
i \frac{d}{d t} \varphi_{k}^{\lambda}(t)=\left[h_{k}+V_{k}(t)\right] \varphi_{k}^{\lambda}(t),
$$

with boundary conditions $\varphi_{k}^{\lambda}(0)=\varphi_{k}^{\lambda}$ [we recall that $h_{k}(z)=$ $h_{k}$ for $z$ on the forward/backward branch; see again Eq. (5)]. The time-dependent HF potential appearing in Eq. (19) can be calculated from Eqs. (6) and (7) by taking into account that for $z=t$ on the forward/backward branch we have $G\left(z, z^{+}\right)=$ $G^{<}(t, t)$.

Let us show that the solution of Eq. (19) is

$$
\varphi_{k}^{\lambda}(t)=e^{-i\left(e_{k}^{\lambda}+\mu-\sigma_{z} \frac{\delta \mu}{2}\right) t} \varphi_{k}^{\lambda},
$$

where $\sigma_{z}$ is the $2 \times 2$ Pauli matrix,

$$
\mu \equiv \frac{\mu_{c}+\mu_{v}}{2}
$$

is the center-of-mass chemical potential, and

$$
\delta \mu \equiv \mu_{c}-\mu_{v}
$$

is the relative one. Inserting Eq. (20) in $G_{k}^{<}$to evaluate the HF potential, we find $V_{k}(t)=e^{i \sigma_{\sigma} \frac{\delta \mu}{2} t} V_{k} e^{-i \sigma_{z} \frac{\delta \mu}{2} t}$. Taking into account that $h_{k}$ is diagonal, we get

$$
\left[h_{k}+V_{k}(t)\right] \varphi_{k}^{\lambda}(t)=e^{-i\left(e_{k}^{\lambda}+\mu-\sigma_{z} \frac{\delta \mu}{2}\right) t}\left[h_{k}+V_{k}\right] \varphi_{k}^{\lambda} .
$$


Next we observe that $h_{k}=h_{k}^{\mathrm{M}}+m$, see Eq. (8), and that the $2 \times 2$ matrix $m$ can be written as $m=\mu \mathbb{1}-\frac{\delta \mu}{2} \sigma_{z}$. Hence

$$
\begin{aligned}
{\left[h_{k}+V_{k}\right] \varphi_{k}^{\lambda} } & =\left[h_{k}^{\mathrm{M}}+V_{k}+\mu \mathbb{1}-\frac{\delta \mu}{2} \sigma_{z}\right] \varphi_{k}^{\lambda} \\
& =\left[e_{k}^{\lambda}+\mu \mathbb{1}-\frac{\delta \mu}{2} \sigma_{z}\right] \varphi_{k}^{\lambda},
\end{aligned}
$$

where in the last row we used that $\varphi_{k}^{\lambda}$ are eigenvectors of $h_{k}^{\mathrm{M}}+$ $V_{k}$. Thus, we can rewrite Eq. (23) as

$$
\left[h_{k}+V_{k}(t)\right] \varphi_{k}^{\lambda}(t)=\left[e_{k}^{\lambda}+\mu \mathbb{1}-\frac{\delta \mu}{2} \sigma_{z}\right] \varphi_{k}^{\lambda}(t),
$$

which coincides with the time derivative $i \frac{d}{d t} \varphi_{k}^{\lambda}(t)$. We emphasize that all quantities needed to evaluate the lesser Green's function have been previously calculated to determine the Matsubara Green's function. The greater Green's function can be derived similarly; the final result looks like Eq. (18) after replacing if $\left(e_{k}^{\lambda}\right) \rightarrow-i \bar{f}\left(e_{k}^{\lambda}\right)$.

Due to the presence of $\sigma_{z}$ in Eq. (20), only the diagonal elements of $G_{k}^{<}$depend on the time-difference. The off-diagonal ones oscillate monochromatically at the frequency $\delta \mu / 2$. In particular, the order parameter $\Delta_{k}(t)=V_{k}^{c v}(t)=\left[V_{k}^{c v}(t)\right]^{*}$ oscillates monochromatically at the frequency $\delta \mu$, i.e.,

$$
\Delta_{k}(t)=\Delta_{k} e^{-i \delta \mu t},
$$

in agreement with Ref. [32]. The time dependence of the order parameter in the NEQ-EI phase resembles the behavior of the superconducting order parameter in a Josephson junction. Josephson oscillations between two superconducting electrodes separated by a thin insulator arise by applying a dc voltage to the equilibrium junction, thus effectively introducing a difference in the electrochemical potentials. This mechanism has recently been investigated by replacing the superconductors with EI electrodes [53-56]. The NEQ-EI oscillations in Eq. (26) are of a slightly different nature. They can be understood in terms of an exotic Josephson junction where Cooper pairs are formed by electrons in different electrodes. In our system, the electrodes are the valence and conduction band and the Cooper pairs are the bound excitons. As we shall see in Sec. VI B, the oscillating behavior of $\Delta_{k}$ has interesting implications in time-resolved photocurrent spectra.

\section{PHASE DIAGRAM}

To illustrate the possible solutions of the self-consistent calculation, we consider the band structure

$$
\epsilon_{\alpha k}=(-)^{\alpha}\left\{2 T[1-\cos (k)]+\epsilon_{g} / 2\right\},
$$

where $(-)^{v} \equiv-1$ and $(-)^{c} \equiv 1, T>0$, and a short-range Coulomb interaction $U_{q}=U$ independent of $q$. The wave vectors $k, q, \ldots$ vary in the first Brillouin zone $(-\pi, \pi)$. Then the HF potential $V_{k}^{\alpha \beta}$ is independent of $k$, see Eqs. (11) and (12), and hence $\Delta_{k}=\Delta$ is independent of $k$ too. We consider the system at zero temperature and express all energies in units of the bare gap $\epsilon_{g}$. We fix the bandwidths $W=4 T=2$, the center-of-mass chemical potential $\mu=0$, and we vary $U \geqslant 0$ and $\delta \mu \geqslant 0$. For $\delta \mu=0$ we recover the ground-state Green's function. Figure 1 shows the color plot of the self-consistent

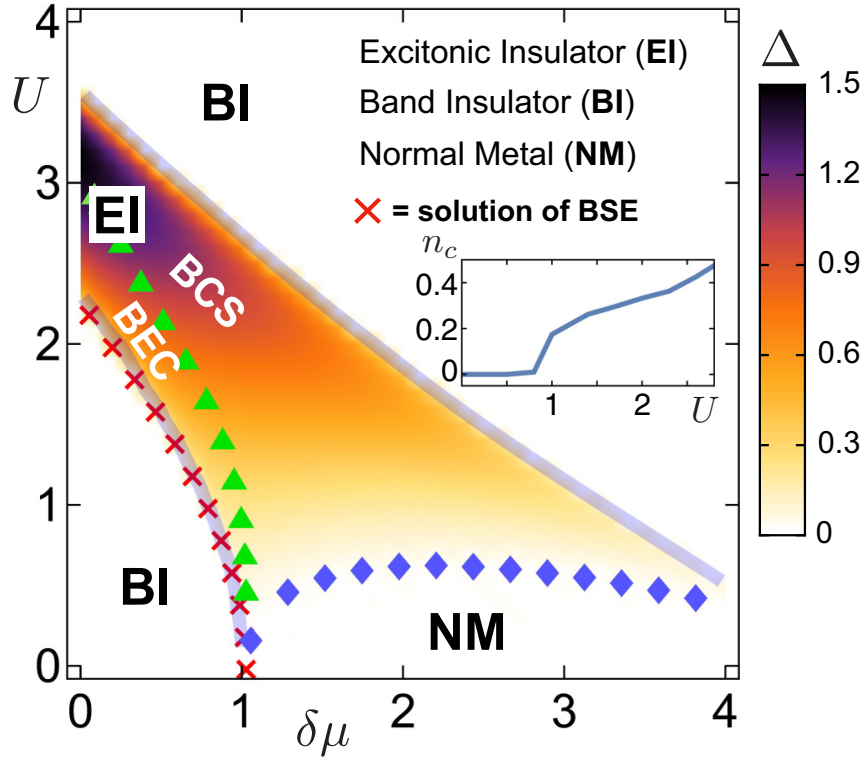

FIG. 1. Color plot of $\Delta$ for different $\delta \mu$ and interaction strength $U$. The values of $\Delta$ have been selected on the basis of the principle of minimum energy. Red crosses at the BI-EI phase boundary correspond to the zero-momentum excitonic poles of the Bethe-Salpeter equation (BSE) with kernel $\delta \Sigma / \delta G$ (for $U=0$ this energy is the bare gap). The BEC-BCS crossover (green triangles) is determined by the condition $\xi=r_{s}$ where $\xi$ is the width of the vanishing-momentum excitonic wave function in real space, and $r_{s}$ is the average distance between electrons in the conduction band. The EI-NM crossover (blue diamonds) is determined by the condition $\Delta<10^{-2}$. The inset shows the values of the conduction density $n_{c}=N_{c} / \mathcal{N}$ vs the interaction $U$ along the BEC-BCS crossover (green triangles). All energies are in units of the bare band gap $\epsilon_{g}$.

$\Delta$. In the white regions we have the trivial (and unique) solution $\Delta=0$. These are the BI regions characterized by $N_{c}=0$ or $N_{v}=0$, and hence by a gap $\epsilon_{g}$ between occupied and unoccupied single-particle states. In the colored region we have two solutions: the trivial one $(\Delta=0)$ and the nontrivial one $(\Delta \neq 0)$. For both solutions, we have estimated the total energy according to [51]

$$
E=-\frac{i}{2} \sum_{k} \int \frac{d \omega}{2 \pi} \operatorname{Tr}\left[\left(\omega+h_{k}\right) G_{k}^{<}(\omega)\right]
$$

and found that the energy of the nontrivial solution is always the lowest. In Sec. III A we calculate the spectral function and show that the unoccupied and occupied single-particle states are separated by an energy gap $\Delta$. Hence the system is still an insulator. However, since $N_{c}$ and $N_{v}$ are both nonvanishing, this insulating phase can only be due to the Coulomb attraction between a conduction electron and a valence hole. We then say that the system is in the EI phase. The EI phase exists in the ground state $(\delta \mu=0)$ for large enough $U$ as well as in excited states $(\delta \mu \neq 0)$ even for rather small $U$ 's. The region below the blue diamonds is characterized by a nontrivial solution with small $\Delta$ (in this region $\Delta \leqslant 10^{-2}$ ); here the system behaves essentially like a normal metal (NM). 

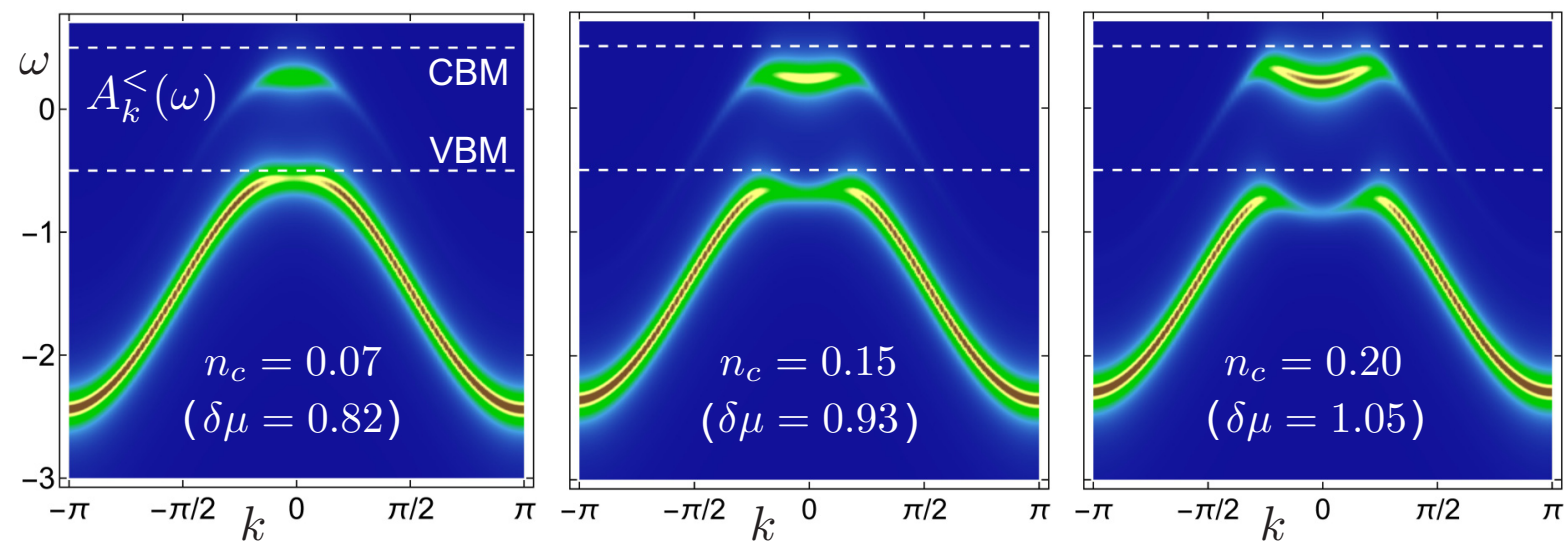

FIG. 2. Occupied part of the spectral function for different values of $\delta \mu$ and $U=1$. Dashed lines indicate the conduction-band minimum $(\mathrm{CBM})$ and valence-band maximum $(\mathrm{VBM})$. All energies are in units of the bare gap $\epsilon_{g}$.

\section{A. Spectral function}

From the NEGF solution of Sec. II B we can extract the occupied part of the spectral function according to

$$
A_{k}^{<}(\omega)=-i \operatorname{Tr}\left[G_{k}^{<}(\omega)\right],
$$

where $G_{k}^{\alpha \alpha,<}(\omega)$ is the Fourier transform of $G_{k}^{\alpha \alpha,<}\left(t, t^{\prime}\right)$ with respect to the time difference. In Fig. 2 we show results for $U=1$ and three different $\delta \mu=0.82,0.93,1.05$ corresponding to a density of electrons in the conduction band $n_{c} \equiv N_{c} / \mathcal{N}=-i \sum_{k} G_{k}^{c c,<}(t, t) / \mathcal{N}=0.07,0.15,0.20$. Notice that for $U=1$ the nontrivial solution $\Delta \neq 0$ exists only for values of $\delta \mu>0.76$; see again Fig. 1. Interestingly, for our parameters the energy of the zero-momentum exciton is $\epsilon_{\mathrm{x}} \simeq 0.76$. The fact that $\epsilon_{\mathrm{x}}$ coincides with the value of $\delta \mu$ at the BI-EI boundary is not a coincidence [37]. In Appendix A we show that the BI-EI boundary (red crosses in Fig. 1) is determined by the excitonic poles of the Bethe-Salpeter equation (BSE) with the kernel given by $\delta \Sigma / \delta G$.

For $\delta \mu=0.82$ (low densities $n_{c}$ ) an excitonic structure with the same shape as the valence band appears at roughly $\epsilon_{g}-\delta \mu$ below the conduction-band minimum (CBM), in agreement with the analytic result in Ref. [57]. The spectral function on the left panel also agrees with the results of Ref. [58], where a system with one single exciton, i.e., $n_{c}=$ $1 / \mathcal{N}$, was considered. By increasing further $\delta \mu$ (and hence the density in the conduction band), the excitonic structure changes its convexity; see the middle and right panels in Fig. 2. This phenomenon is distinct from the one reported in Ref. [58], where the change of convexity is obtained by averaging spectral functions each with one single exciton of different center-of-mass momentum. In our case, the convexto-concave shape transition develops with increasing the density of excitons; see also Sec. III B.

The unoccupied part of the spectral function can be calculated similarly: $A_{k}^{>}(\omega)=-i \operatorname{Tr}\left[G_{k}^{>}(\omega)\right]$. Since $\mu=0$, the system is particle-hole-symmetric and therefore

$$
A_{k}^{>}(\omega)=A_{k}^{<}(-\omega) \text {. }
$$

As anticipated, there is a gap of order $\Delta$ between the unoccupied and occupied bands.

\section{B. BEC-BCS crossover}

The EI phase is similar to the BCS superconducting phase when the width $\xi$ of the excitons is larger than the average electron distance (or Wigner-Seitz radius) $r_{s}$. In the opposite regime, i.e., $\xi \ll r_{s}$, the excitons behave like pointlike bosons in a Bose-Einstein condensate (BEC). To determine the BEC-BCS crossover [7,37,49,59], we have calculated the width $\xi$ of the zero-momentum excitonic wave function and we compare it with the average distance $r_{s}$ of electrons in the conduction band. The excitonic wave function in real space is given by (modulo a normalization constant) $Y(x)=$ $\sum_{k} e^{i k x} Y_{k}$, with $Y_{k}$ the excitonic solution of Eq. (A4), whereas (for one-dimensional systems) $r_{s} \simeq \mathcal{N} / N_{c}=1 / n_{c}$. The green triangles in the phase diagram correspond to the values of $U$ and $\delta \mu$ for which $\xi=r_{s}$, where $\xi$ has been estimated from $Y(\xi) / Y(0)=0.1$. The inset of Fig. 1 shows $n_{c}$ versus $U$ when we move along the BEC-BCS crossover line (green triangles). The curve represents the values of $n_{c}$ above which, for that given $U$, the NEQ-EI changes from a BEC to a BCS condensate; as expected, $n_{c}$ increases with increasing $U$.

An alternative way to find the values of $\delta \mu$ at the BEC-BCS crossover consists in determining the onset of the broadening of the excitonic population in momentum space-in the $\mathrm{BEC}$ regime, the excitonic population is peaked around zero momentum. Let us write the many-body state of the system in the NEQ-EI phase. Since the minus branch $e_{k}^{-}$is entirely below the plus branch $e_{k}^{+}$we have

$$
\left|\Psi_{\mathrm{x}}\right\rangle=\prod_{p}\left(\varphi_{v p}^{-} \hat{v}_{p}^{\dagger}+\varphi_{c p}^{-} \hat{c}_{p}^{\dagger}\right)|0\rangle,
$$

where $|0\rangle$ is the state with no electrons. We define the creation operator for an exciton of momentum $q$ according to

$$
\hat{b}_{q}^{\dagger}=\sum_{k} Y_{k}^{(q)} \hat{c}_{k+q}^{\dagger} \hat{v}_{k},
$$

where the excitonic amplitude $Y_{k}^{(q)}$ can be calculated by solving the analog of Eq. (A4) for finite-momentum excitons. It is a matter of simple algebra to show that

$$
\left\langle\Psi_{\mathrm{x}}\left|\hat{c}_{k^{\prime}+q^{\prime}}^{\dagger} \hat{v}_{k^{\prime}} \hat{v}_{k}^{\dagger} \hat{c}_{k+q}\right| \Psi_{\mathrm{x}}\right\rangle=\delta_{q q^{\prime}} \delta_{k k^{\prime}}\left(\varphi_{c k}^{-} \varphi_{c k+q}^{-}\right)^{2}
$$




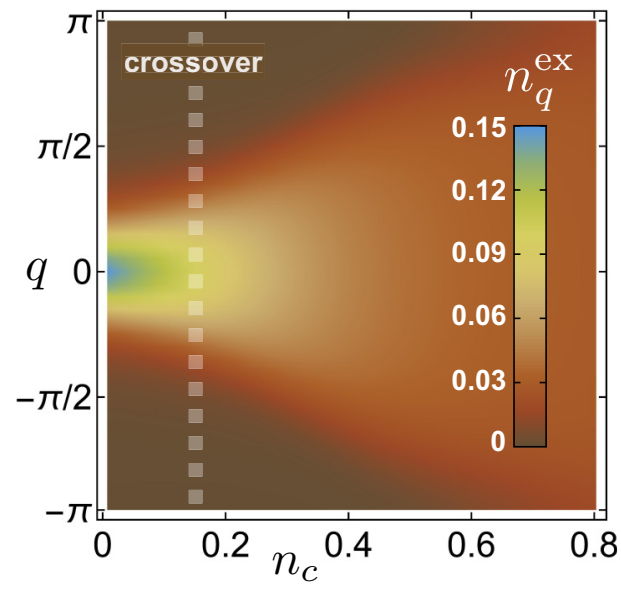

FIG. 3. Color plot of the excitonic population in momentum space for different densities $n_{c}=N_{c} / \mathcal{N}=1 / r_{s}$ for $U=1$.

Therefore, the excitonic density in the NEQ-EI phase is given by

$$
n_{q}^{\mathrm{ex}} \equiv\left\langle\Psi_{\mathrm{x}}\left|\hat{b}_{q}^{\dagger} \hat{b}_{q}\right| \Psi_{\mathrm{x}}\right\rangle=\sum_{k}\left(Y_{k}^{(q)} \varphi_{c k}^{-} \varphi_{c k+q}^{-}\right)^{2}
$$

In Fig. 3 we show $n_{q}^{\text {ex }}$ for the interaction strength $U=1$ and different densities in the conduction band. The broadening begins when $n_{c}=N_{c} / \mathcal{N}=1 / r_{s} \simeq 0.15$. This density is obtained when $\delta \mu \simeq 0.93$, in agreement with the abscissa of the green triangle in Fig. 1 for the same value of $U$.

Remarkably, the convex-to-concave shape transition in the excitonic structure of the spectral function roughly occurs at the BEC-BCS crossover. In Fig. 2 the Coulomb strength is $U=1$ and the transition occurs for $\delta \mu \simeq 0.93$, in agreement with the previously estimated value. We have verified (not shown) that this property remains true for all values of $U<U_{c} \simeq 2.3$ (for larger $U$ 's the ground state is an EI). We infer that time-resolved ARPES spectra may provide a tool to detect the BEC-BCS crossover in NEQ-EI's; see also Sec. VI A.

\section{LiF BULK INSULATOR}

We now apply the NEGF theory for excited states, see Sec. II, to a LiF bulk insulator. In the ground state this material has an experimental gap $\epsilon_{g} \simeq 14.5 \mathrm{eV}$ and the optical spectrum exhibits a $1 s$ bright exciton with a binding energy of about $1.9 \mathrm{eV}$ [60]. The $1 s$ exciton is threefold degenerate due to the cubic symmetry of the system. The degenerate multiplet can therefore be labeled by the three orthogonal directions of the excitonic dipole moment.

The goal of the present section is to demonstrate that the results obtained in the two-band model are also found in a real material when multiple bands and valleys, intraband and interband repulsion, band anisotropies and degeneracies, as well as spin-exchange effects are all taken into account. We first compute the phase diagram of $\mathrm{LiF}$ and then construct the spectral function in the NEQ-EI phase.

\section{A. Phase diagram}

In a first-principles calculation, the band energies and Bloch states depend on the single-particle formalism (DFT, mean-field theory, etc.) and on the approximation made within the formalism. The Bloch states provide a basis to write the exact many-body Hamiltonian $\hat{H}$ and to define the number operator $\hat{N}_{c}$ of conduction electrons. In general, $\hat{N}_{c}$ does not commute with $\hat{H}$ whatever the formalism and approximation are. Thus, the Hamiltonian of $\mathrm{LiF}$ (or any other insulator) is not invariant under the gauge transformation of Sec. II, i.e., $\hat{c} \rightarrow e^{i \theta} \hat{c}$. Nevertheless, a properly chosen single-particle basis will still prove to be a convenient way to understand the properties of the NEQ-EI phase since the Coulomb-driven recombination-time of particle-hole excitations is longer than any other timescales in the considered low-energy sector (i.e., in the Hilbert space of weakly excited states). This is equivalent to saying that the commutator $\left[\hat{H}, \hat{N}_{c}\right]$ is negligible and hence that the gauge symmetry is almost exact.

Another difference between the model system of Sec. II and $\mathrm{LiF}$ is the interparticle interaction. In the former case, the interaction is short-ranged and the self-energy can be treated in the HF approximation. Instead, in $\mathrm{LiF}$ we have to deal with the long-ranged nature of the Coulomb interaction. Due to the importance of screening effects, we evaluate the self-energy in the Hartree plus screened exchange (HSEX) approximation, $\Sigma=\Sigma_{\mathrm{HSEX}}$, which consists in replacing the bare interaction of the Fock term with a statically screened RPA interaction $W$. The RPA $W$ is calculated using first-shot ground-state HSEX quasiparticle energies (RPA@HSEX) and it is kept fixed in all self-consistent calculations, i.e., we ignore the dependence of $W$ on the electronic populations (or equivalently on $\delta \mu$ ). This is justified in the BEC regime up to the BEC-BCS crossover since the excitonic contribution to the screening is much smaller than that of free carriers and the exciton density is small. Details regarding the calculation of $W$, the self-consistent procedure, and the numerical implementation can be found in Appendix B.

One key feature of the NEQ-EI phase is the relation between the exciton energy and the border of the BI-EI transition. In the HSEX approximation, the exciton energy is, by construction, given by the poles of the Bethe-Salpeter equation (BSE) with (i) HSEX single-particle energies and wave functions for the bare electron-hole propagator, and (ii) $\delta \Sigma_{\mathrm{HSEX}} / \delta G$ for the kernel (BSE@HSEX). Notice that the functional derivative $\delta W / \delta G$ appearing in the kernel vanishes since $W$ is fixed. The BSE@HSEX is different from stateof-the-art BSE implementations where LDA single-particle energies and wave functions are used for both the bare electron-hole propagator and the calculation of the RPA $W$ (BSE@LDA). Quasiparticle corrections are included in the bare electron-hole propagator only. To assess the quality of the proposed RPA@HSEX screening, we have preliminarily solved the BSE@HSEX and compared the resulting absorption spectrum with the experimental one from Ref. [60] and with the one resulting from a standard implementation of the BSE@LDA [61]; see Fig. 4. The agreement between BSE@HSEX and the experiment is of comparable quality to a BSE@LDA calculation. In fact, we found that the HSEX wave functions, being more localized than LDA ones, reduce the intensity of the bare electron-hole interaction, but this 


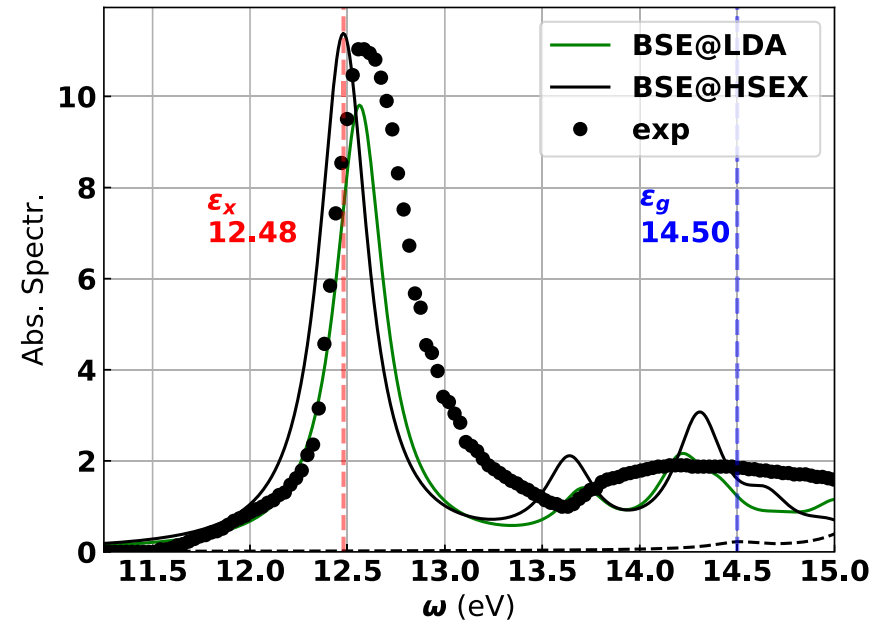

FIG. 4. The absorption spectra (in arbitrary units) of LiF resulting from BSE@HSEX (solid black) and BSE@LDA [61] (solid green) is compared to experimental data [60] (dotted black). The vertical dashed lines are drawn at the exciton energy $\epsilon_{\mathrm{x}}=12.48 \mathrm{eV}$ (red) and quasiparticle gap $\epsilon_{g}=14.5 \mathrm{eV}$ (blue).

reduction is compensated by the use of HSEX energies in the RPA $W$. In particular, the $1 s$ excitonic peak is located at about $\epsilon_{\mathrm{x}}=12.48 \mathrm{eV}$ with respect to the valence-band maximum (VBM), hence its binding energy is correctly $\sim 2 \mathrm{eV}$.

Self-consistent HSEX calculations with $\delta \mu>0$ have been carried out using an initial Green's function with off-diagonal elements in the (ground-state) HSEX basis, yielding a small macroscopic polarization along one of the principal axes. All momenta in the first Brillouin zone have been considered independent, i.e., no spatial symmetry has been imposed. The HSEX basis provides a good reference basis to construct an approximate order parameter. In fact, at self-consistency and for $\delta \mu=0$ both the Green's function $G_{\mathbf{k}}^{\alpha \beta,<}\left(t, t^{\prime}\right)$ and the single-particle HSEX Hamiltonian $h_{\mathbf{k}}^{\alpha \beta, \operatorname{HSEX}}\left[G^{<}(t, t)\right]$ (a functional of the equal-time $G^{<}$through $\Sigma^{\mathrm{HSEX}}$ ) are diagonal in this basis. We define the approximate order parameter as the following zero-momentum $(\mathbf{k}=\mathbf{0}=\Gamma)$ average:

$$
\Delta_{0}=\left|\sum_{c, v} h_{\mathbf{k}=\mathbf{0}}^{c v, \text { HSEX }}\right|,
$$

where the sum extends over all valence $(v)$ and conduction (c) bands. Equation (35) reduces to Eq. (15) in the two-band model if the HSEX Hamiltonian is replaced by the HF one. In Fig. 5 we show the order parameter $\Delta_{0}$ versus $\delta \mu$. Below the exciton energy $\epsilon_{\mathrm{x}}$ the HSEX Hamiltonian remains diagonal and the order parameter $\Delta_{0}=0$. A symmetry breaking clearly occurs in the NEQ-EI phase $\left(\delta \mu>\epsilon_{\mathrm{x}}\right)$, and it is characterized by a sharp increase of $\Delta_{0}$ near $\delta \mu=\epsilon_{\mathrm{x}}$.

To further characterize the NEQ-EI phase in LiF, we have also calculated the macroscopic polarization $\mathbf{P}$ versus $\delta \mu$; see again Fig. 5. As expected, $\mathbf{P}$ becomes nonzero right at $\delta \mu=\epsilon_{\mathrm{x}}$ since the single-exciton state has a nonvanishing dipole matrix element with the ground state. We observe that any NEQ-EI state with $|\mathbf{P}| \neq \mathbf{0}$ is at least sixfold degenerate $( \pm|\mathbf{P}|$ along three orthogonal axis) due to the cubic symmetry of LiF. Thus, the cubic symmetry and the gauge symmetry break

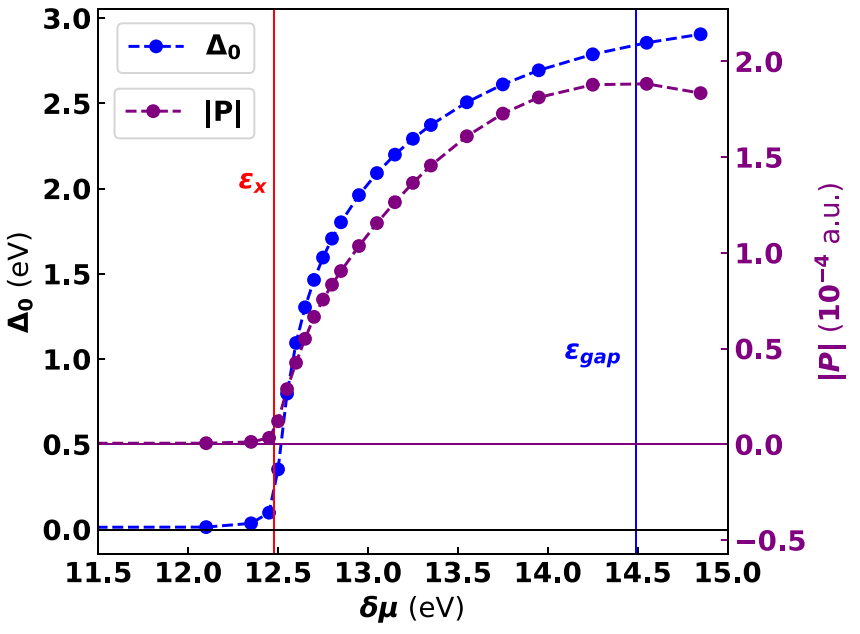

FIG. 5. Order parameter (blue) and macroscopic polarization (purple) vs $\delta \mu$ for a bulk LiF. The zero of energy is the VBM. The vertical lines are drawn at the exciton energy $\epsilon_{\mathrm{x}}=12.48 \mathrm{eV}$ (red) and quasiparticle gap $\epsilon_{g}=14.5 \mathrm{eV}$ (blue).

for the same values of $\delta \mu$. It is also worth mentioning that if the system were described by a statistical mixture of the six degenerate states, all with equal weights, then the cubic symmetry would be recovered (since the average of $\mathbf{P}$ would be zero), but $\Delta_{0}$ would remain finite, it being independent of the polarization direction. The same situation would occur if the system were described by a state with only (nondegenerate) dark excitons. Neither the dark-excitonic state nor the statistical mixture can be realized with a laser pulse, which naturally breaks the symmetry through the polarization of the electric field. On the contrary, the single polarized selfconsistent solution with bright $1 s$ excitons can, in principle, be generated by a suitable laser pulse; see Sec. V.

\section{B. Spectral function}

We now compute the occupied part of the spectral function $A_{\mathbf{k}}^{<}(\omega)=-i \operatorname{Tr}\left[G_{\mathbf{k}}^{<}(\omega)\right]$ in the NEQ-EI phase. Let us define $|n \mathbf{k}\rangle$ and $\epsilon_{n \mathbf{k}}$ as the single-particle states and energies that diagonalize the ground-state HSEX Hamiltonian $(\delta \mu=0)$. Similarly, we define $|\tilde{n} \mathbf{k}\rangle$ and $\epsilon_{\tilde{n} \mathbf{k}}$ as the single-particle states and energies that diagonalize the excited-state HSEX Hamiltonian $(\delta \mu>0)$. The explicit expressions for $G_{\mathbf{k}}^{n n,<}(\omega)$ read

$$
\begin{aligned}
& G_{\mathbf{k}}^{c c,<}(\omega)=2 \pi i \sum_{\tilde{v}} \delta\left(\omega-\epsilon_{\tilde{v} \mathbf{k}}-\delta \mu / 2\right)|\langle c \mathbf{k} \mid \tilde{v} \mathbf{k}\rangle|^{2}, \\
& G_{\mathbf{k}}^{v v,<}(\omega)=2 \pi i \sum_{\tilde{v}} \delta\left(\omega-\epsilon_{\tilde{v} \mathbf{k}}+\delta \mu / 2\right)|\langle v \mathbf{k} \mid \tilde{v} \mathbf{k}\rangle|^{2} .
\end{aligned}
$$

As long as $\delta \mu \leqslant \epsilon_{\mathrm{x}},\langle c \mathbf{k} \mid \tilde{v} \mathbf{k}\rangle=0$ and only the valence bands contribute to $A_{\mathbf{k}}^{<}(\omega)$. For $\delta \mu \gtrsim \epsilon_{\mathrm{x}}$ (low-density limit) we have $\epsilon_{\tilde{v} \mathbf{k}} \approx \epsilon_{v \mathbf{k}},\langle\tilde{v} \mathbf{k} \mid v \mathbf{k}\rangle \approx 1$, and $|\langle c \mathbf{k} \mid \tilde{v} \mathbf{k}\rangle|^{2} \propto\left|Y_{c v, \mathbf{k}}\right|^{2}$ with $Y_{c v, \mathbf{k}}$ the $1 s$ excitonic wave function; compare Eq. (31) with Eq. (A3). Therefore, the conduction states contribute to $A_{\mathbf{k}}^{<}(\omega)$ by replicating the valence-band structure at an energy $\delta \mu$ higher and with a weight proportional to $\left|Y_{c v, \mathbf{k}}\right|^{2}$ [57].

In Fig. 6 we display the spectral function for three different values of $n_{c}$ (conduction electrons per unit cell) and thus for three different values of $\delta \mu$. The ground-state $(\delta \mu=0)$ values 

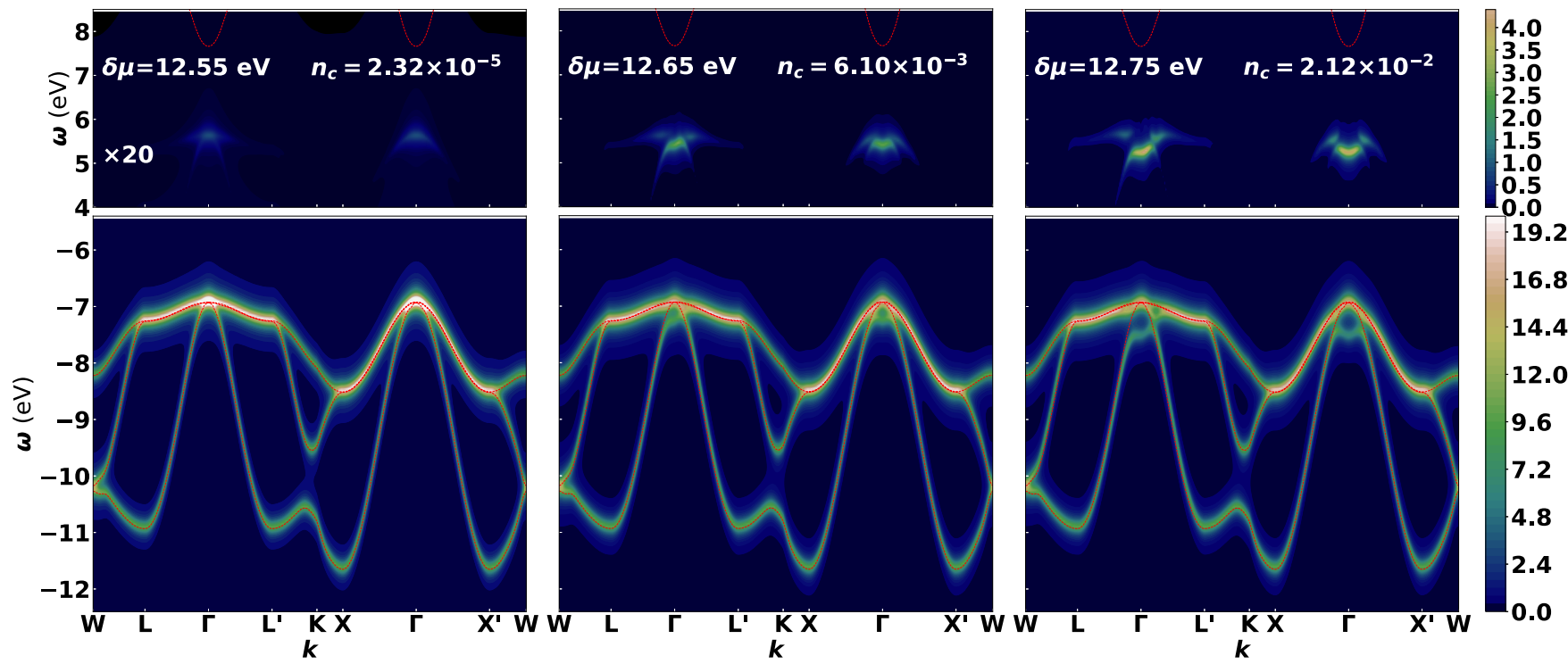

FIG. 6. Occupied part of the spectral function of a bulk LiF for different values of $\delta \mu$. Energy (vertical axis) is measured with respect to the middle of the equilibrium gap and momentum (horizontal axis) runs along the closed path $W-L-\Gamma-L^{\prime}-K-X-\Gamma-X^{\prime}-W$ of the first Brillouin zone The values of the chemical potential and of the resulting density of conduction electrons per unit cell are reported in the figure. At positive energy, the signal has been scaled up by a factor of 20 . The red dashed lines represent the equilibrium band structure.

are displayed with dashed red lines and show that the band structure is threefold degenerate at $\Gamma$ (consistently with the degeneracy of the $1 s$ exciton). In the low-density limit (left panel), the degeneracy is almost preserved and, as expected, the contribution of the conduction states is just a replica of the valence band. As the density increases (middle and right panels), the degeneracy at the $\Gamma$ point manifestly breaks. The intensity along the equivalent direction in the Brillouin zone is different; see, for instance, the paths $\Gamma \rightarrow L$ and $\Gamma \rightarrow L^{\prime}$, where $L$ and $L^{\prime}$ are connected by the symmetry operations of the crystal at equilibrium. Even more pronounced than the asymmetric intensity is the asymmetry in the energy dispersion. Interestingly, the most dramatic change involves the lowest-energy valence band with the other two valence bands mainly playing the role of spectators. This scenario is very similar to that of the two-band model. In both cases, the shape of the excitonic structure resembles locally (close to the $\Gamma$ point) the shape of the valence bands, and upon increasing the population in the conduction band, the maximum at $\Gamma$ turns into a minimum causing the excitonic structure to undergo the convex-to-concave shape transition; see Figs. 2 and 6 (middle and right panel).

The results presented in this section support the use of the two-band model for understanding other general features of the NEQ-EI phase.

\section{PUMP-DRIVEN BI-EI TRANSITION}

In this section, we demonstrate that the self-consistent NEQ-EI phase is accessible by shining suitable laser pulses on the BI ground state. All results in this section refer to the two-band model in the HF approximation.

We consider again the Hamiltonian of Eq. (1) at zero temperature and set $\delta \mu=0$ (ground-state) and $U<U_{c}$ (BI phase with $\Delta=0$ ). The system is driven out of equilib- rium by a time-dependent electric field $E(t)$ coupled to the valence-conduction dipole moments $D_{k}$. The driving Hamiltonian reads

$$
\hat{H}_{\text {drive }}(t)=E(t) \sum_{k} D_{k}\left(\hat{c}_{k}^{\dagger} \hat{v}_{k}+\hat{v}_{k}^{\dagger} \hat{c}_{k}\right)
$$

This light-matter coupling has been used to study changes in the optical properties due to a strong monochromatic electric field (dynamical Stark effects) [62,63] and more recently weak monochromatic fields such as those generated in quantum optical cavities [64]. Here we are interested in the electronic properties of the system when the electric field is a pump-pulse centered around frequency $\omega_{P}$ with finite duration $T_{P}$ :

$$
E(t)=\theta\left(1-\left|1-2 t / T_{P}\right|\right) E_{P} \sin ^{2}\left(\frac{\pi t}{T_{P}}\right) \sin \left(\omega_{P} t\right)
$$

Henceforth we consider the interaction strength $U=1$, dispersion of Eq. (27) with bandwidth $W=4 T=2$, center-ofmass chemical potential $\mu=0$, and, for simplicity, dipole moments $D_{k}=D$ independent of $k$ (energies are in units of the bare gap $\epsilon_{g}$ ). The real-time HF simulations have been performed with the CHEERS code [65], and times are expressed in units of $1 / \epsilon_{g}$. Since the driving Hamiltonian depends on $E_{P}$ and $D$ only through their product, we define the Rabi frequency $\Omega_{P} \equiv E_{P} D$.

The main result of the simulations is that for $\omega_{P}$ larger than the exciton energy $\epsilon_{\mathrm{x}}$, see Eq. (A4), and smaller than $\epsilon_{g}$, the modulus of the order parameter attains a constant and finite value after the pump, in agreement with Ref. [44]. In Fig. 7(a), we show the steady values of the density in the conduction band $n_{c}$ and of the modulus of the order parameter $|\Delta|$ versus the pump frequency $\omega_{P}$ (we recall that for the chosen parameters $\epsilon_{\mathrm{x}}=0.76$ ) for a pump pulse of duration $T_{P}=100$ and Rabi frequency $\Omega_{P}=0.05$; see Fig. 7(d). Independently 

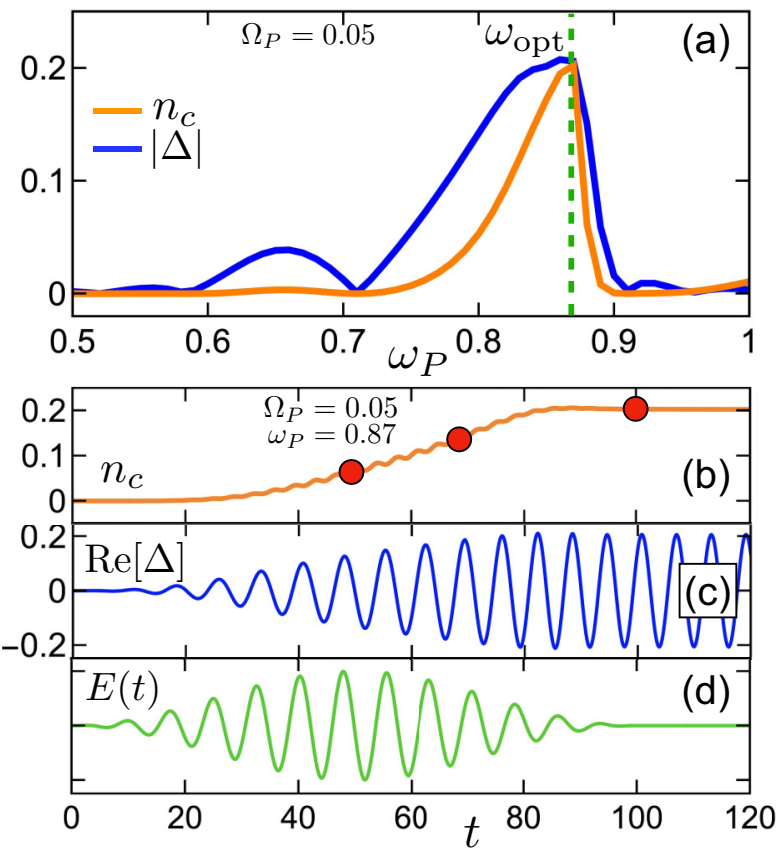

FIG. 7. (a) Density in the conduction band $n_{c}$ and modulus of the order parameter $|\Delta|$ after the pump vs the pump frequency $\omega_{P}$. Time-dependent density in the conduction band (b), real part of the order parameter (c), and pump-pulse profile (d). The red dots in panel (b) are drawn at the values of $n_{c}=0.07,0.15,0.20$. Energies are in units of $\epsilon_{g}$ and times in units of $1 / \epsilon_{g}$.

of the values of $T_{P}$ and $\Omega_{P}$, the trend of Fig. 7(a) is general: there exists an optimal frequency $\omega_{\text {opt }} \in\left(\epsilon_{\mathrm{x}}, \epsilon_{g}\right)$ (of course depending on $T_{P}$ and $\Omega_{P}$ ) at which the steady values of $n_{c}$ and $|\Delta|$ are simultaneously maximized. We point out that in the noninteracting case, $U=0$, the steady value of $|\Delta|$ is zero for all $\omega_{P}, T_{P}$, and $\Omega_{P}$ due to perfect dephasing.

In Figs. 7(b) and 7(c) we show $n_{c}(t)$ and $\operatorname{Re}[\Delta(t)]$ during the action of the pump pulse with $\omega_{P}=\omega_{\mathrm{opt}} \simeq 0.87$. We observe the occurrence of persistent monochromatic oscillations in $\operatorname{Re}[\Delta(t)]$ after $T_{P}=100$. We therefore conclude that

$$
\Delta\left(t>T_{P}\right)=e^{-i \omega_{E} t}|\Delta|,
$$

where $\omega_{E}$ is a pump-dependent frequency. The timedependent behavior of the order parameter is the same as that of the self-consistent solution; see Eq. (26). We have extracted the frequency $\omega_{E}$ from the real-time solution $\operatorname{Re}[\Delta(t)]$, set $\delta \mu=\omega_{E}$ in the self-consistent calculation of Sec. II A, and found that the self-consistent values of $n_{c}$ and $\Delta$ are identical to the steady-state values of $n_{c}$ and $|\Delta|$. This remains true for several different pump intensities (not shown). We therefore conclude that the pump-driven state is precisely the NEQ-EI state analyzed in detail in Sec. II.

A second piece of evidence in favor of our conclusion is presented in Fig. 8, where we show the values of $\omega_{E}$ (empty circle) resulting from pumping at the optimal frequency (black triangle) for $T_{P}=100$ and different Rabi frequencies $\Omega_{P}$. In the limit of vanishing $\Omega_{P}$ (hence vanishing intensities of the electric field), the density pumped in the conduction band approaches zero and both $\omega_{E}$ and $\omega_{\text {opt }}$ approach the energy $\epsilon_{\mathrm{x}}=0.76$ of the zero-momentum exciton (dashed

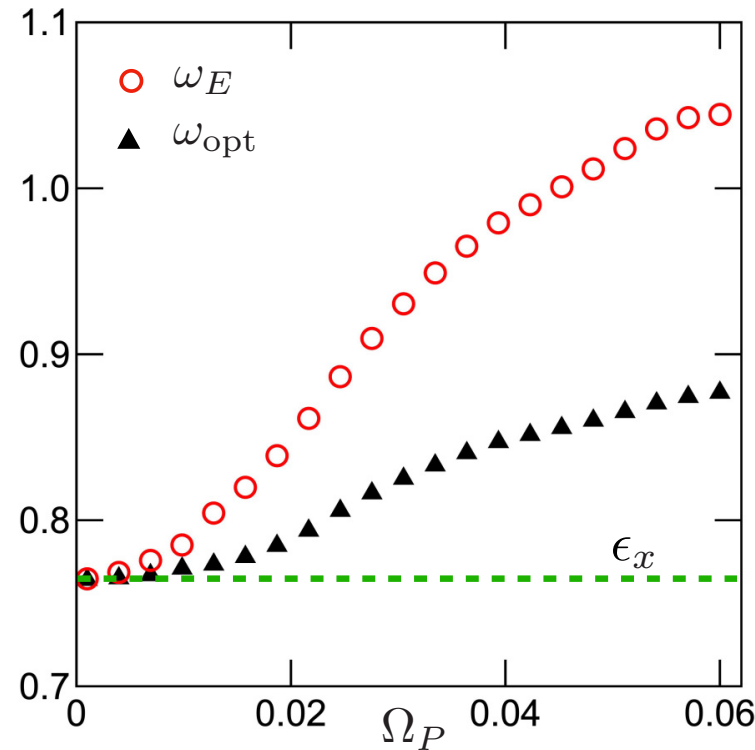

FIG. 8. Frequency $\omega_{E}$ (empty circle) resulting from pumping at the optimal frequency (black triangle) for different Rabi frequencies $\Omega_{P}$. The value of the excitonic energy $\epsilon_{\mathrm{x}}=0.76$ is highlighted with a dashed green line. All energies are in units of $\epsilon_{g}$.

green line). This is the same behavior of $\delta \mu$ versus $n_{c}$. Indeed, a vanishingly small value of $n_{c}$ in the self-consistent solution implies a vanishingly small value of $\Delta$, which we know to occur for $\delta \mu=\epsilon_{\mathrm{x}}$; see Appendix A.

\section{TIME-RESOLVED ARPES}

The time-resolved ARPES signal is proportional to the number of electrons $N_{k}(\omega)$ with energy $\omega$ and parallel momentum $k$ ejected by a probing pulse $\mathbf{e}(t)$. For arbitrary probe pulses, we have $[57,66]$

$$
N_{k}(\omega)=2 \sum_{\alpha \beta} \int d t d \bar{t} \operatorname{Re}\left[\Sigma_{k, \omega}^{\alpha \beta, \mathrm{R}}(t, \bar{t}) G_{k}^{\beta \alpha,<}(\bar{t}, t)\right],
$$

where the ionization self-energy reads

$$
\Sigma_{k, \omega}^{\alpha \beta, \mathrm{R}}(t, \bar{t})=-i \theta(t-\bar{t})\left[\mathbf{D}_{k, \omega}^{\alpha} \cdot \mathbf{e}(t)\right] e^{-i \omega(t-\bar{t})}\left[\mathbf{D}_{k, \omega}^{\beta} \cdot \mathbf{e}(\bar{t})\right]
$$

and $\mathbf{D}_{k, \omega}^{\alpha}$ is the dipole matrix element between a band state $\alpha k$ and a continuum LEED state of energy $\omega$ and parallel momentum $k$.

\section{A. BEC-BCS crossover with femtosecond probe pulses}

From Eq. (41) we see that $N_{k}(\omega)$ is a complicated twotimes convolution of $G^{<}\left(t, t^{\prime}\right)$. Let us introduce the relative time $\tau \equiv t-t^{\prime}$ and the center-of-mass time $t_{\mathrm{CM}} \equiv\left(t+t^{\prime}\right) / 2$. Since the lesser Green's function yields the probability amplitude for a hole to propagate freely from $t^{\prime}$ to $t$, the $G^{<}\left(t, t^{\prime}\right)$ varies in $\tau$ no slower than the inverse of the energy of the highest occupied state. For a system in equilibrium this energy is the ionization potential; in our nonequilibrium system, this energy can be estimated as the CBM, i.e., $\epsilon_{c 0}$. The timescale of the variation of $G^{<}$in the center-of-mass time $t_{\mathrm{CM}}$ can be inferred from the rate of change of the occupations, see 

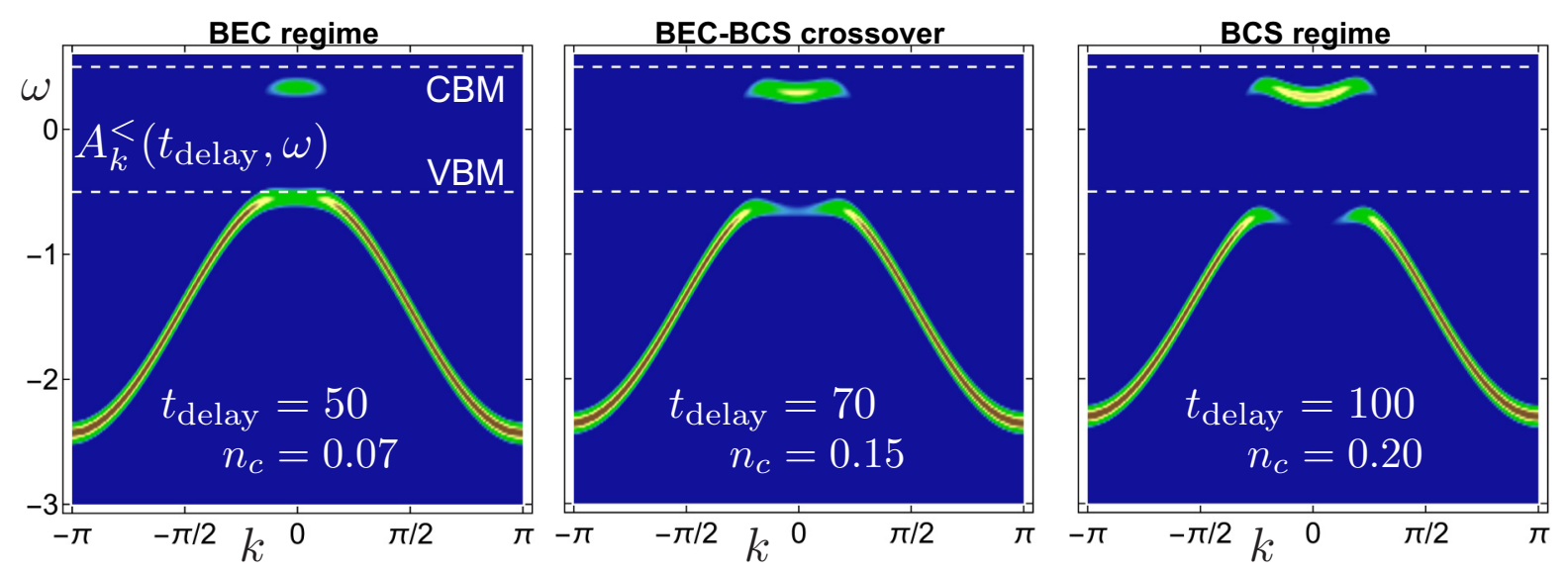

FIG. 9. Occupied part of the transient spectral function for different delays corresponding to a density in the conduction band $n_{c}=$ $0.07,0.15,0.20$. Energies are in units of $\epsilon_{g}$ and times in units of $1 / \epsilon_{g}$.

Fig. 7(b), and can be estimated as a fraction of the pump duration $T_{P}$. For band gaps in the $\mathrm{eV}$ range, our pump pulse has a duration $T_{P} \simeq 10^{2}$ fs. Therefore, a probe pulse of duration $T_{p} \simeq T_{P} / 10 \simeq 10^{1}$ fs and central frequency $\omega_{p} \gg 2 \pi / T_{p}$ is enough to resolve all removal energies provided that $T_{p} \gg$ $2 \pi / \epsilon_{c 0}$. If such a probe impinges on the system at time $t_{\text {delay }}$, then $N_{k}(\omega) \propto A_{k}^{<}\left(t_{\text {delay }}, \omega-\omega_{p}\right)$, where the transient spectral function is defined according to

$$
A^{<}\left(t_{\text {delay }}, \omega\right)=-i \int d \tau e^{i \omega \tau} \operatorname{Tr}\left[G^{<}\left(t, t^{\prime}\right)\right]
$$

and $t_{\text {delay }}=t_{\mathrm{CM}}$. Of course, for $t_{\text {delay }} \gg T_{P}$ the transient spectral function becomes independent of $t_{\text {delay }}$.

To obtain $A^{<}\left(t_{\text {delay }}, \omega\right)$ we observe that the time-dependent HF equations give access to the "time diagonal" $G_{k}^{<}(t, t)$ from which we can calculate the retarded HF Green's function,

$$
G_{k}^{\mathrm{R}}\left(t, t^{\prime}\right)=-i \theta\left(t, t^{\prime}\right) \mathcal{T}\left\{e^{-i \int_{t^{\prime}}^{t} d \bar{t}\left[h_{k}+D_{k} E(\bar{t}) \sigma_{x}+V_{k}(\bar{t})\right]}\right\},
$$

where $\sigma_{x}$ is the Pauli matrix; see Eq. (38). From $G_{k}^{\mathrm{R}}\left(t, t^{\prime}\right)=$ $\left[G_{k}^{\mathrm{A}}\left(t^{\prime}, t\right)\right]^{*}$ we can extract the "time off-diagonal" $G_{k}^{<}\left(t, t^{\prime}\right)$ as [51]

$$
G_{k}^{<}\left(t, t^{\prime}\right)=-i G_{k}^{\mathrm{R}}\left(t, t^{\prime}\right) G_{k}^{<}\left(t^{\prime}, t^{\prime}\right)+i G_{k}^{<}(t, t) G^{\mathrm{A}}\left(t, t^{\prime}\right),
$$

and calculate the transient spectral function from Eq. (43).

We consider again the pump pulse in Fig. 7(d). The green dots are drawn at times $t=50,70,100$ for which $n_{c}=$ $0.07,0.15,0.20$, respectively; these values of the conduction density are the same as in the three panels of Fig. 2. In Fig. 9 we show the color plot of $A^{<}\left(t_{\text {delay }}, \omega\right)$ at $t_{\text {delay }}=50,70,100$. For $t_{\text {delay }} \lesssim T_{P}$ the pump is still active and the transient spectral function is, in general, affected by nonadiabatic effects [67]. In our case, these are very small and the agreement between Figs. 2 and 9 is unexpectedly good. Incidentally, we observe that this agreement supports the adiabatic approximation employed in Ref. [57] to calculate time-resolved ARPES spectra. We also point out that the trace in Eq. (43) involves only the diagonal elements of $G^{<}$, which, in accordance with Eq. (18), depend only on the time difference. Accordingly, replicas of the band structure $[68,69]$ do not occur. The absence of replicas also follows from a corollary of the Floquet theorem according to which a monochromatic driving that couples two orthogonal subspaces (valence and conduction states in our case) do not introduce a time dependence in the average of operators acting on one of the two subspaces [70]. For $t_{\text {delay }} \gg T_{P}$, the transient spectral function becomes independent of $t_{\text {delay }}$ (as it should be) and indistinguishable from the right panel of Fig. 2 (not shown). This fact further corroborates the equivalence between the pump-driven state and the NEQ-EI state of Sec. II.

From Fig. 9 we also see that the convex-to-concave shape transition of the excitonic structure (signaling the BEC-BCS crossover) can be revealed by time-resolved ARPES spectra. It is worth emphasizing that linear-response theory, i.e., $n_{c} \propto \Omega_{P}^{2}$, is totally inadequate to describe the pump-driven evolution of the system toward the BCS regime. In Fig. 10 we show the time-dependent evolution of $n_{c}(t)$ for $\omega_{P}=0.87$,

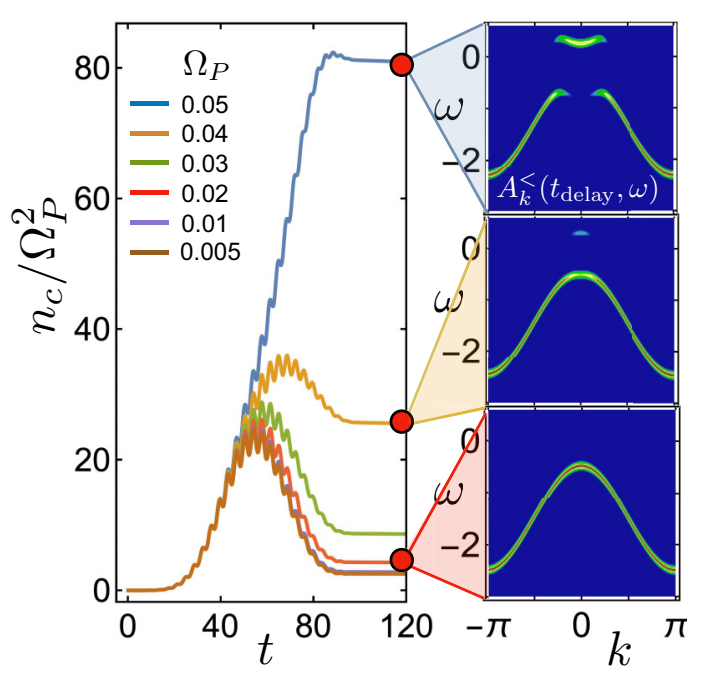

FIG. 10. Time-dependent density in the conduction band for pump pulses of duration $T_{P}=100$, central frequency $\omega_{P}=0.87$, and different Rabi frequencies $\Omega_{P}$. The right panels show the steady-state spectral function calculated at $t_{\text {delay }}=110$ for $\Omega_{P}=$ $0.02,0.04,0.05$. Energies are in units of $\epsilon_{g}$ and times in units of $1 / e_{g}$. 
$T_{P}=100$, and different values of the Rabi frequency $\Omega_{P}$. For $\Omega_{P}=0.02,0.04,0.05$ we display the corresponding steadystate $\left(t_{\text {delay }} \gg T_{P}\right)$ spectral functions. The concavity changes for $\Omega_{P}=0.05$, and for this Rabi frequency the steady density of conduction electrons is clearly not proportional to $\Omega_{P}^{2}$.

\section{B. Josephson oscillations with attosecond probe pulses}

The ARPES signal changes dramatically for ultrashort probe pulses since the probe has no time to wash out the oscillatory contribution of the off-diagonal $G^{<}$. To highlight the main qualitative difference, we consider a $\delta$-like pulse of the form

$$
\mathbf{e}(t)=\mathbf{e}_{0} \delta\left(t-t_{\text {delay }}\right) .
$$

Then, the number of electrons in Eq. (41) becomes

$$
N_{k}(\omega)=-\sum_{\alpha \beta} \operatorname{Im}\left[\Omega_{k, \omega}^{\alpha} \Omega_{k, \omega}^{\beta} G_{k}^{\beta \alpha,<}\left(t_{\text {delay }}, t_{\text {delay }}\right)\right],
$$

where we have defined the Rabi frequencies

$$
\Omega_{k, \omega}^{\alpha}=\mathbf{D}_{k, \omega}^{\alpha} \cdot \mathbf{e}_{0} .
$$

At the steady state, $G_{k}^{\alpha \alpha,<}(t, t)$ is independent of $t$ but $G_{k}^{c v,<}(t, t)=-\left[G_{k}^{v c,<}(t, t)\right]^{*} \sim e^{-i \delta \mu t}$; see Eq. (18). Consequently, $N_{k}(\omega)$ consists of a dc part $N_{k}^{(\mathrm{dc})}(\omega)$ and an ac part of amplitude $N_{k}^{(\mathrm{ac})}(\omega)$ and frequency $\delta \mu$ :

$$
N_{k}(\omega)=N_{k}^{(\mathrm{dc})}(\omega)+N_{k}^{(\mathrm{ac})}(\omega) \sin \left[(\delta \mu) t_{\text {delay }}+\varphi\right] .
$$

We therefore predict oscillations in $N_{k}(\omega)$ versus $t_{\text {delay }}$ even for $t_{\text {delay }} \gg T_{P}$. These oscillations should be observed in timeresolved ARPES provided that the duration of the probe pulse is much smaller than the period $2 \pi / \delta \mu$. For values of $\delta \mu$ in the $\mathrm{eV}$ range (like in $\mathrm{LiF}$ ), probe durations of the order of a hundred attoseconds are sufficient.

The ac response in Eq. (49) can again be explained using the analogy with the exotic Josephson junction; see Sec. II B. The application of a pump pulse coupling electrons in different electrodes (bands in our case) is equivalent to the application of a dc bias between the electrodes. If the $\mathrm{dc}$ bias is kept on for a time $T_{P}$, then a macroscopic number of electrons is transferred from one electrode (valence band) to the other (conduction band), thus generating a difference in the electrochemical potentials of the electrodes. Once the dc bias (pump in our case) is switched off, this difference does not damp to zero since electrons cannot hop back to the original electrode (band). This difference in electrochemical potentials is equivalent to the difference $\delta \mu$ discussed in Sec. II.

\section{SUMMARY AND OUTLOOK}

We presented a derivation of the NEQ-EI phase using the NEGF formalism on the Konstantinov-Perel' contour. The NEQ-EI phase can be physically induced by pump pulses with properly chosen subgap frequency, and the nature (BEC or $\mathrm{BCS}$ ) of the excitonic condensate can be tuned by the pump duration. The most remarkable difference between a groundstate EI and a NEQ-EI is the time-dependent behavior of the order parameter and the total polarization. In fact, they both exhibit persistent, self-sustained monochromatic oscillations even at vanishing pump. Interestingly, these oscillations have the same nature of the ac oscillations in an exotic Josephson junction, where Cooper pairs are formed by electrons at the opposite side of the junction.

The NEQ-EI phase leaves clear fingerprints in timeresolved ARPES spectra. For probe durations long enough to resolve the band structure, the oscillatory contribution of the order parameter is washed out and an excitonic "band" appears inside the gap. Depending on the BEC or BCS nature of the excitonic condensate, this band can be either convex or concave. Time-resolved ARPES experiments could in principle monitor the convex-to-concave shape transition using, e.g., pump pulses of hundreds of femtoseconds and overlapping probe pulses of a few femtoseconds shone at different delays. Ultrafast probe pulses of, e.g., a few hundred attoseconds do instead broaden the band structure and unveil the time-dependent contribution. The resulting photocurrent oscillates in time even after the end of the pump. The period of the oscillations is the same as the period of the order parameter, which is no smaller than the exciton energy $\epsilon_{\mathrm{x}}$.

The equivalence between the time-dependent approach and the self-consistent NEGF scheme for excited states allows for studying the NEQ-EI phase in realistic materials using different strategies. The inclusion of electronic correlations at, e.g., the $G W$ level is more feasible in the self-consistent scheme. On the other hand, the effects of phonon-induced coherence losses and exciton recombination on the self-sustained oscillations of the total polarization could be investigated using the real-time Kadanoff-Baym equations [51] in the GKBA framework [71]. Of course accurate calculations are needed for quantitative predictions on specific materials. However, the highlighted qualitative features of the NEQ-EI phase are general and provide a useful guide for the interpretation of time-resolved ARPES spectra.

\section{ACKNOWLEDGMENTS}

G.S. and E.P. acknowledge funding through the RISE Co-ExAN (Grant No. GA644076) and the INFN17_nemesys project. G.S., D.S., and E.P. acknowledge funding through the MIUR PRIN (Grant No. 20173B72NB). A.M., D.S., and E.P. acknowledge funding through the European Union project MaX Materials design at the eXascale H2020-EINFRA-20151 (Grants Agreement No. 676598 and No. 824143) and Nanoscience Foundries and Fine Analysis-Europe H2020INFRAIA-2014-2015 (Grant Agreement No. 654360). G.S. acknowledges Tor Vergata University for financial support through the Mission Sustainability Project 2DUTOPI.

\section{APPENDIX A: BETHE-SALPETER EQUATION AND THE BI-EI PHASE BOUNDARY}

The equation for the BI-EI boundary marked with red crosses in Fig. 1 can be obtained analytically. Infinitesimally close to the boundary, $\Delta_{k}$ is small. Then the minus band $e_{k}^{-}$is entirely below the plus band $e_{k}^{+}$and only $\lambda=-$ contributes in the sum of Eq. (12). Expanding Eq. (17) to lowest order in $\Delta_{k}$ and taking into account that $\tilde{\epsilon}_{v k}-\tilde{\epsilon}_{c k}<0$, we find

$$
\varphi_{v k}^{-} \simeq-1, \quad \varphi_{c k}^{-} \simeq-\frac{\Delta_{k}}{\tilde{\epsilon}_{v k}-\tilde{\epsilon}_{c k}} .
$$


Using this expansion, the diagonal elements of the HF potential can be approximated to first order in $\Delta_{k}$ as $V^{v v} \simeq 0$ and $V_{k}^{c c} \simeq U_{0}$; see Eq. (11). Consequently, from Eqs. (13) and (14) we get $\tilde{\epsilon}_{v k} \simeq \epsilon_{v k}-\mu_{v}$ and $\tilde{\epsilon}_{c k} \simeq \epsilon_{c k}-\mu_{c}$, which implies that $\tilde{\epsilon}_{v k}-\tilde{\epsilon}_{c k}=\epsilon_{v k}-\epsilon_{c k}+\delta \mu$. Using this result in Eq. (A1), inserting the resulting expressions into Eq. (12), and recalling that, by the definition in Eq. (15), $V_{k}^{v c}=\Delta_{k}$, we get

$$
\Delta_{k}=-\frac{1}{\mathcal{N}} \sum_{q} U_{k-q} \frac{\Delta_{q}}{\epsilon_{v q}-\epsilon_{c q}+\delta \mu} .
$$

The lowest value of $\delta \mu$ for which this equation admits nontrivial solutions defines the BI-EI boundary.

Interestingly, Eq. (A2) coincides with the Bethe-Salpeter equation (BSE) for the zero-momentum response function. For time-local kernels, the BSE is indeed equivalent to the eigenvalue equation of a many-body eigenstate with a single exciton of vanishing momentum. Let $\left|\Psi_{0}\right\rangle=\prod_{k} \hat{v}_{k}^{\dagger}|0\rangle$ be the ground state of energy $E_{0}$ in the BI phase, and let us write the one-exciton state as

$$
\left|\Psi_{\mathrm{x}}\right\rangle=\sum_{k} Y_{k} \hat{c}_{k}^{\dagger} \hat{v}_{k}\left|\Psi_{0}\right\rangle=\sum_{k} Y_{k}\left|\Phi_{k}\right\rangle,
$$

where we introduced the $e h$ states $\left|\Phi_{k}\right\rangle \equiv \hat{c}_{k}^{\dagger} \hat{v}_{k}\left|\Psi_{0}\right\rangle$. Since $\hat{H}$ preserves the number of electrons in each band, $\hat{H}\left|\Psi_{\mathrm{x}}\right\rangle$ is again a linear combination of the $\left|\Phi_{k}\right\rangle$ 's. The possible excited-state energies $E=E_{0}+\epsilon_{\mathrm{x}}$ are found by solving the eigenvalue problem $\hat{H}\left|\Psi_{\mathrm{x}}\right\rangle=\left(E_{0}+\epsilon_{\mathrm{x}}\right)\left|\Psi_{\mathrm{x}}\right\rangle$. Inserting the expansion in Eq. (A3) and taking the sandwich with $\left\langle\Phi_{k}\right|$ yields

$$
\left(\epsilon_{c k}-\epsilon_{v k}-\epsilon_{\mathrm{x}}\right) Y_{k}=\frac{1}{\mathcal{N}} \sum_{q} U_{k-q} Y_{q} .
$$

Renaming $\epsilon_{\mathrm{x}}=\delta \mu$ and setting $Y_{q}=\Delta_{q} /\left(\epsilon_{v q}-\epsilon_{c q}+\delta \mu\right)$, we see by inspection that Eq. (A4) is identical to Eq. (A2). Thus, for a given $U$ the BI-EI boundary is found at $\delta \mu=\epsilon_{\mathrm{x}}$, in agreement with Ref. [37]. For $U=0$, the BI-EI boundary is at $\delta \mu=\epsilon_{g}$, as it should be, whereas for $U \geqslant U_{c} \simeq 2.3$ (in this case the ground state is an EI) the solutions of Eq. (A2) occur for $\delta \mu \leqslant 0$. The $\delta \mu \leqslant 0$ criterion is indeed used to establish the occurrence of an equilibrium EI phase [5].

\section{APPENDIX B: IMPLEMENTATION DETAILS FOR LIF CALCULATIONS}

We have determined the Kohn-Sham basis by performing an LDA self-consistent calculation with the QUANTUMESPRESSO package [72]. The ground state has been converged using a $6 \times 6 \times 6, \Gamma$ centered grid with a cutoff of $80 \mathrm{Ry}$. Then the wave functions have been computed with a non-selfconsistent calculation on a $16 \times 16 \times 16$ grid. Our equilibrium bands well reproduce those of Ref. [73] with a KS gap of $8.45 \mathrm{eV}$, which is quite far from the experimental value. To improve the description, we have constructed the Hartree plus screened exchange (HSEX) potential using the YAMBO code [47]. The implementation writes the Hamiltonian to be solved self-consistently in the basis set of the KS wave functions. This makes it possible to include nonlocal (in space) selfenergies like HSEX. The SEX contribution to the self-energy is obtained by replacing the bare interaction $v$ in the Fock term with the static RPA screened interaction $W=v\left(1+\chi^{\mathrm{RPA}} v\right)$, where $\chi^{\mathrm{RPA}}$ is the solution of the Dyson equation $\chi^{\mathrm{RPA}}=$ $\chi^{0}+\chi^{0} v \chi^{\mathrm{RPA}}$ and (in real-space coordinates)

$$
\chi^{0}\left(\mathbf{r}, \mathbf{r}^{\prime}\right)=\sum_{\mu}^{\text {occ unocc }} \sum_{\nu} \sum_{\mathbf{k} \mathbf{k}^{\prime}} \frac{\varphi_{\nu \mathbf{k}^{\prime}}(\mathbf{r}) \varphi_{\mu \mathbf{k}}^{*}(\mathbf{r}) \varphi_{\nu \mathbf{k}^{\prime}}^{*}\left(\mathbf{r}^{\prime}\right) \varphi_{\mu \mathbf{k}}\left(\mathbf{r}^{\prime}\right)}{\epsilon_{\mu \mathbf{k}}-\epsilon_{\nu \mathbf{k}^{\prime}}}
$$

The RPA response function $\chi^{\mathrm{RPA}}=\chi^{\mathrm{RPA}}[\varphi, \epsilon]$ is a functional of the single-particle wave functions $\varphi$ and energies $\epsilon$. A first approximated screened interaction $W^{(0)}$ has been evaluated using LDA wave functions and energies, i.e., $\chi^{\mathrm{RPA}}=$ $\chi^{\mathrm{RPA}}\left[\varphi^{\mathrm{LDA}}, \epsilon^{\mathrm{LDA}}\right]$. The self-consistent HSEX spectrum is found to be well approximated by the original $\mathrm{KS}$ spectrum if the KS valence (conduction) energies are stretched by a factor 1.65 (1.4). The stretched KS energies with a scissor of $6 \mathrm{eV}$ (necessary to reach the experimental gap) have been used to improve $\chi^{\mathrm{RPA}}=\chi^{\mathrm{RPA}}\left[\varphi^{\mathrm{LDA}}, \epsilon_{\text {stretched }+ \text { scissor }}^{\mathrm{LDA}}\right]$ and hence the screened interaction. We verified that a further iteration of the screened interaction did not change substantially the stretching factors. The improved interaction, henceforth denoted by $W^{(1)}$, widens the gap of the self-consistent HSEX spectrum from 8.5 to $12.8 \mathrm{eV}$; we have therefore applied a scissor of $1.7 \mathrm{eV}$ to match the experimental value of the gap. The equilibrium bands are displayed in Fig. 6 with dashed red lines. We mention that our HSEX self-energy is close to the Hartree plus Coulomb-hole(COH)-SEX self-energy used in the literature in a number of work [74-79].

The interaction $W^{(1)}$ has also been used to solve the BetheSalpeter equation with a bare HSEX electron-hole propagator (BSE@HSEX). The agreement with the experimental optical spectrum is of comparable quality as state-of-the-art BSE implementations; see Fig. 4. To obtain the correct values of the HSEX dipoles, also including the commutator with the nonlocal SEX self-energy, the covariant approach recently implemented in YAMBO [80] has been used.

To be consistent with the equilibrium calculation where the screened interaction $W$ is fixed to $W^{(1)}$, we have carried out the self-consistent NEQ Matsubara procedure of Sec. II using a fixed $W=W^{(1)}$ in the Fock term. For both equilibrium and NEQ calculations, we have explicitly considered 50 bands. The screened interaction and the screened contribution to the exchange term has been calculated with $8 \mathrm{Ry}(=59$ reciprocallattice vectors), whereas the Hartree term and the unscreened contribution to the exchange term have been calculated with 64 Ry (965 reciprocal-lattice vectors). In the self-consistency cycles, we have fixed the total charge per unit cell (charge neutrality) instead of the center-of-mass chemical potential $\mu$, and convergence is established on the single-particle energies. All parameters, i.e., energy cutoff, number of bands, number of grid points, etc., have been monitored; the converged energies have an error smaller than $0.1 \mathrm{eV}$.
[1] J. M. Blatt, K. W. Böer, and W. Brandt, Phys. Rev. 126, 1691 (1962).
[2] L. V. Keldysh and Y. U. Kopaev, Fiz. Tverd. Tela. 6, 2791 (1964) [Sov. Phys. Solid State 6, 2219 (1965)]. 
[3] A. N. Kozlov and L. A. Maksimov, J. Exptl. Theor. Phys. (U.S.S.R.) 48, 1184 (1965) [JETP 21, 790 (1965)].

[4] D. Jérome, T. M. Rice, and W. Kohn, Phys. Rev. 158, 462 (1967).

[5] B. Halperin and T. Rice, Solid State Phys. 21, 115 (1968).

[6] L. V. Keldysh and A. N. Kozlov, Zh. Eksp. Teor. Fiz. 54, 978 (1968) [JETP 27, 521 (1968)].

[7] C. Comte and P. Nozières, J. Phys. (France) 43, 1069 (1982).

[8] I. B. Spielman, J. P. Eisenstein, L. N. Pfeiffer, and K. W. West, Phys. Rev. Lett. 87, 036803 (2001).

[9] J. P. Eisenstein and A. H. MacDonald, Nature (London) 432, 691 (2004)

[10] C.-H. Zhang and Y. N. Joglekar, Phys. Rev. B 77, 233405 (2008).

[11] H. Min, R. Bistritzer, J.-J. Su, and A. H. MacDonald, Phys. Rev. B 78, 121401(R) (2008).

[12] Y. E. Lozovik and A. A. Sokolik, JETP Lett. 87, 55 (2008).

[13] M. Y. Kharitonov and K. B. Efetov, Phys. Rev. B 78, 241401(R) (2008).

[14] D. Varsano, S. Sorella, D. Sangalli, M. Barborini, S. Corni, E. Molinari, and M. Rontani, Nat. Commun. 8, 1461 (2017).

[15] M. Hellgren, J. Baima, and A. Acheche, Phys. Rev. B 98, 201103(R) (2018).

[16] H. Cercellier, C. Monney, F. Clerc, C. Battaglia, L. Despont, M. G. Garnier, H. Beck, P. Aebi, L. Patthey, H. Berger et al., Phys. Rev. Lett. 99, 146403 (2007).

[17] C. Monney, G. Monney, P. Aebi, and H. Beck, New J. Phys. 14, 075026 (2012).

[18] B. Zenker, H. Fehske, H. Beck, C. Monney, and A. R. Bishop, Phys. Rev. B 88, 075138 (2013).

[19] B. Zenker, H. Fehske, and H. Beck, Phys. Rev. B 90, 195118 (2014).

[20] A. Kogar, M. S. Rak, S. Vig, A. A. Husain, F. Flicker, Y. I. Joe, L. Venema, G. J. MacDougall, T. C. Chiang, E. Fradkin et al., Science 358, 1314 (2017).

[21] D. Golež, P. Werner, and M. Eckstein, Phys. Rev. B 94, 035121 (2016).

[22] Y. Murakami, D. Golež, M. Eckstein, and P. Werner, Phys. Rev. Lett. 119, 247601 (2017).

[23] S. Mor, M. Herzog, D. Golež, P. Werner, M. Eckstein, N. Katayama, M. Nohara, H. Takagi, T. Mizokawa, C. Monney et al., Phys. Rev. Lett. 119, 086401 (2017).

[24] D. Werdehausen, T. Takayama, M. Höppner, G. Albrecht, A. W. Rost, Y. Lu, D. Manske, H. Takagi, and S. Kaiser, Sci. Adv. 4, eaap8652 (2018).

[25] D. Werdehausen, T. Takayama, G. Albrecht, Y. Lu, H. Takagi, and S. Kaiser, J. Phys.: Condens. Matter 30, 305602 (2018).

[26] T. Tanabe, K. Sugimoto, and Y. Ohta, Phys. Rev. B 98, 235127 (2018).

[27] R. Tuovinen, D. Golez, M. Schler, P. Werner, M. Eckstein, and M. A. Sentef, Phys. Status Solidi B 256, 1800469 (2019).

[28] Y. Wakisaka, T. Sudayama, K. Takubo, T. Mizokawa, M. Arita, H. Namatame, M. Taniguchi, N. Katayama, M. Nohara, and H. Takagi, Phys. Rev. Lett. 103, 026402 (2009).

[29] K. Seki, Y. Wakisaka, T. Kaneko, T. Toriyama, T. Konishi, T. Sudayama, N. L. Saini, M. Arita, H. Namatame, M. Taniguchi et al., Phys. Rev. B 90, 155116 (2014).

[30] C. Triola, A. Pertsova, R. S. Markiewicz, and A. V. Balatsky, Phys. Rev. B 95, 205410 (2017).

[31] A. Pertsova and A. V. Balatsky, Phys. Rev. B 97, 075109 (2018).
[32] M. H. Szymańska, J. Keeling, and P. B. Littlewood, Phys. Rev. Lett. 96, 230602 (2006).

[33] R. Hanai, P. B. Littlewood, and Y. Ohashi, J. Low Temp. Phys. 183, 127 (2016).

[34] R. Hanai, P. B. Littlewood, and Y. Ohashi, Phys. Rev. B 96 , 125206 (2017).

[35] R. Hanai, P. B. Littlewood, and Y. Ohashi, Phys. Rev. B 97, 245302 (2018).

[36] K. W. Becker, H. Fehske, and V.-N. Phan, Phys. Rev. B 99, 035304 (2019).

[37] M. Yamaguchi, K. Kamide, T. Ogawa, and Y. Yamamoto, New J. Phys. 14, 065001 (2012).

[38] M. Yamaguchi, K. Kamide, R. Nii, T. Ogawa, and Y Yamamoto, Phys. Rev. Lett. 111, 026404 (2013).

[39] S. Schmitt-Rink, D. S. Chemla, and H. Haug, Phys. Rev. B 37, 941 (1988).

[40] H. Haug, J. Lumin. 30, 171 (1985).

[41] S. Glutsch and R. Zimmermann, Phys. Rev. B 45, 5857 (1992).

[42] H. Chu and Y. C. Chang, Phys. Rev. B 54, 5020 (1996).

[43] Y. Murotani, C. Kim, H. Akiyama, L. N. Pfeiffer, K. W. West, and R. Shimano, Phys. Rev. Lett. 123, 197401 (2019).

[44] T. Östreich and K. Schönhammer, Z. Phys. B 91, 189 (1993).

[45] X. Jun, Z. Mervin, W. Yuan, and Z. Xiang, Nanophotonics 6, 1309 (2017).

[46] K. Hannewald, S. Glutsch, and F. Bechstedt, J. Phys.: Condens. Matter 13, 275 (2000).

[47] A. Marini, C. Hogan, M. Grüning, and D. Varsano, Comput. Phys. Commun. 180, 1392 (2009).

[48] D. M. Eagles, Phys. Rev. 186, 456 (1969).

[49] G. C. Strinati, P. Pieri, G. Röpke, P. Schuck, and M. Urban, Phys. Rep. 738, 1 (2018).

[50] Z.-h. Yang, Y. Li, and C. A. Ullrich, J. Chem. Phys. 137, 014513 (2012).

[51] G. Stefanucci and R. van Leeuwen, Nonequilibrium ManyBody Theory of Quantum Systems: A Modern Introduction (Cambridge University Press, Cambridge, 2013).

[52] O. V. Konstantinov and V. I. Perel', J. Exptl. Theor. Phys. (U.S.S.R.) 39, 197 (1960) [Sov. Phys. JETP 12, 142 (1961)].

[53] M. Rontani and L. J. Sham, Phys. Rev. B 80, 075309 (2009).

[54] Y. N. Joglekar, A. V. Balatsky, and M. P. Lilly, Phys. Rev. B 72, 205313 (2005).

[55] Y.-F. Hsu and J.-J. Su, Sci. Rep. 5, 15796 (2015).

[56] V. Apinyan and T. K. Kopeć, J. Low Temp. Phys. 194, 325 (2019).

[57] E. Perfetto, D. Sangalli, A. Marini, and G. Stefanucci, Phys. Rev. B 94, 245303 (2016).

[58] A. Rustagi and A. F. Kemper, Phys. Rev. B 97, 235310 (2018).

[59] P. Nozières and S. Schmitt-Rink, J. Low Temp. Phys. 59, 195 (1985).

[60] D. M. Roessler and W. C. Walker, J. Opt. Soc. Am. 57, 835 (1967).

[61] M. Rohlfing and S. G. Louie, Phys. Rev. Lett. 81, 2312 (1998).

[62] C. Comte and G. Mahler, Phys. Rev. B 34, 7164 (1986).

[63] C. Comte and G. Mahler, Phys. Rev. B 38, 10517 (1988).

[64] S. Latini, E. Ronca, U. De Giovannini, H. Hübener, and A. Rubio, Nano Lett. 19, 3473 (2019).

[65] E. Perfetto and G. Stefanucci, J. Phys.: Condens. Matter 30, 465901 (2018).

[66] J. K. Freericks, H. R. Krishnamurthy, and T. Pruschke, Phys. Rev. Lett. 102, 136401 (2009). 
[67] P. Myöhänen, A. Stan, G. Stefanucci, and R. van Leeuwen, J. Phys.: Conf. Ser. 220, 012017 (2010).

[68] Y. H. Wang, H. Steinberg, P. Jarillo-Herrero, and N. Gedik, Science 342, 453 (2013).

[69] H. Hübener, U. De Giovannini, and A. Rubio, Nano Lett. 18, 1535 (2018).

[70] E. Perfetto and G. Stefanucci, Phys. Rev. A 91, 033416 (2015).

[71] P. Lipavský, V. Špička, and B. Velický, Phys. Rev. B 34, 6933 (1986).

[72] P. Giannozzi, S. Baroni, N. Bonini, M. Calandra, R. Car, C. Cavazzoni, D. Ceresoli, G. L. Chiarotti, M. Cococcioni, I. Dabo et al., J. Phys.: Condens. Matter 21, 395502 (2009).

[73] N.-P. Wang, M. Rohlfing, P. Krüger, and J. Pollmann, Phys. Rev. B 67, 115111 (2003).

[74] F. Bruneval, N. Vast, and L. Reining, Phys. Rev. B 74, 045102 (2006).
[75] M. Gatti, F. Bruneval, V. Olevano, and L. Reining, Phys. Rev. Lett. 99, 266402 (2007).

[76] J. Vidal, F. Trani, F. Bruneval, M. A. L. Marques, and S. Botti, Phys. Rev. Lett. 104, 136401 (2010).

[77] D. Wegkamp, M. Herzog, L. Xian, M. Gatti, P. Cudazzo, C. L. McGahan, R. E. Marvel, R. F. Haglund, A Rubio, M. Wolf et al., Phys. Rev. Lett. 113, 216401 (2014).

[78] M. Gatti, F. Sottile, and L. Reining, Phys. Rev. B 91, 195137 (2015).

[79] S. Körbel, D. Kammerlander, R. Sarmiento-Pérez, C. Attaccalite, M. A. L. Marques, and S. Botti, Phys. Rev. B 91, 075134 (2015).

[80] D. Sangalli, A. Ferretti, H. Miranda, C. Attaccalite, I. Marri, E. Cannuccia, P. Melo, M. Marsili, F. Paleari, A. Marrazzo et al., J. Phys.: Condens. Matter 31, 325902 (2019). 